\title{
Quantum dynamics of charged and neutral magnetic solitons: Spin-charge separation in the one-dimensional Hubbard model
}

\author{
Mona Berciu and Sajeev John \\ Department of Physics, University of Toronto, 60 St. George Street, Toronto, Ontario, M5S 1A7, Canada
}

(November 25, 2017)

Nos. 71.27.+a, 71.10.Fd, 74.20.Mn

We demonstrate that the Configuration Interaction (CI) Approximation recaptures essential features of the exact (Bethe Ansatz) solution to the 1D Hubbard model. As such, it provides valuable route for describing effects which go beyond mean-field theory for strongly correlated electron systems in higher dimensions. The CI method systematically describes fluctuation and quantum tunneling corrections to the Hartree-Fock Approximation (HFA). HFA predicts that doping a half-filled Hubbard chain leads to the appearance of charged spin-polarons or charged domain-wall solitons in the antiferromagnetic (AFM) background. The CI method, on the other hand, describes the quantum dynamics of these charged magnetic solitons and quantum tunneling effects between various mean-field configurations. In this paper, we test the accuracy of the CI method against the exact solution of the one-dimensional Hubbard model. We find remarkable agreement between the energy of the mobile charged bosonic domain-wall (as given by the CI method) and the exact energy of the doping hole (as given by the Bethe Ansatz) for the entire $U / t$ range. The CI method also leads to a clear demonstration of the spin-charge separation in 1D. Addition of one doping hole to the half-filled antiferromagnetic chain results in the appearance of two different carriers: a charged bosonic domain-wall (which carries the charge but no spin) and a neutral spin-1/2 domain wall (which carries the spin but no charge). 


\section{INTRODUCTION}

The two-dimensional (2D) Hubbard model (or a modified version of it) is widely believed to describe the essential physics of the strongly correlated electrons in high temperature superconducting cuprates.1 Unlike the 1D Hubbard model, an exact solution of the 2D problem is not known, and one must resort to approximations. The validity of such approximations can be tested on the 1D Hubbard model, which is exactly solvable 2 using the Bethe Ansatz 3 It is highly desirable to develop an approximation which recaptures essential physical features of the exact Bethe Ansatz solution and which, at the same time, can be applied to higher dimensional systems.

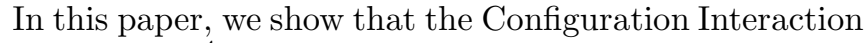
(CI) Method 1 is such an approximation. We demonstrate that the CI method recaptures essential quantum tunneling effects that go beyond mean-field theory and that lead to spin-charge separation in $1 \mathrm{D}$. The predictions of the CI method in the $2 \mathrm{D}$ case as well as a comparison to cyprate superconductors are described in detail elsewhere.5

We consider the generalized Hubbard Hamiltonian:

$$
\mathcal{H}=-t \sum_{\substack{\langle i, j\rangle \\ \alpha \beta}}\left(c_{i \alpha}^{\dagger} T_{\alpha \beta}^{i j} c_{j \beta}+\text { h.c. }\right)+U \sum_{i} c_{i \uparrow}^{\dagger} c_{i \uparrow} c_{i \downarrow}^{\dagger} c_{i \downarrow}
$$

Here, $c_{i \alpha}^{\dagger}$ is the creation operator for an electron of spin $\alpha$ at site $i$, and the notation $\langle i, j\rangle$ means that the sum is restricted to nearest-neighbors sites $i$ and $j$. The parameters of the problem are the hopping matrix $t$, the onsite interaction matrix $U$, and the $\mathrm{SU}(2)$ matrices $T_{\alpha \beta}^{i j}$, which describe phase factors and internal spin rotations acquired by the electron as it hops between sites. For the 1D Hubbard Hamiltonian which we consider in this paper we simply set $T_{\alpha \beta}^{i j}=\delta_{\alpha \beta}$. However, for the $2 \mathrm{D}$ system, it has been shown that a non-trivial choice of the matrices $T_{\alpha \beta}^{i j}$, which describes a $2 \pi$ (internal) spin rotation of the electron as it encircles an elementary plaquette of the square lattice, may be essential to the cuprate physics 6 , For this reason, we develop the CI formalism for the more general Hamiltonian (1).

In addition to providing a test to gauge the accuracy of the Configuration Interaction Method, the 1D results may be directly relevant to certain high $\mathrm{T}_{c}$ cuprate superconductors. $\mathrm{YBa}_{2} \mathrm{Cu}_{3} \mathrm{O}_{7}$ and its close relatives have quasi-one-dimensional $\mathrm{CuO}$ chain structures. Experiments measuring the dc resistivity $\mathrm{E}$, the infrared and optical conducticity 9 and the penetration depth in untwinned crystal 10 and ceramics 11 have revealed large anisotropies between the a-direction (perpendicular to chains) and the b-direction (parallel to chains). These results suggest that substantial currents are carried along the chains in both the normal and superconducting state. The source of superconducting condensate on the chains has not yet been elucidated.

We begin Section 2 with a brief review of the Static Hartree-Fock Approximation (HFA). The HFA leads to a mean-field ground-state with properties which are in disagreement with those of the exact ground-state. For the half-filled (undoped) chain, HFA predicts a degenerate ground-state with long-range antiferromagnetic (AFM) order and with staggered magnetic moments aligned along some arbitrary direction. Even in the presence of a weak external interaction which creates an easy axis for spin orientation, the HF ground state is degenerate with the related mean-field in which all the spins have been flipped $(S \rightarrow-S)$. On the other hand, the true groundstate of the half-filled system is non-degenerate and has no long-range order, despite the presence of strong AFM correlations. When holes are introduced into the chain, the HFA leads to the creation of static magnetic spinpolaron and domain-wall solitons which trap the hole. Since these solitons are motionless in the static HFA, this mean field solution breaks the translational invariance of the original Hamiltonian. The CI method facilitates the restoration of both of the above symmetries in accord with the exact Bethe ansatz ground state.

The essence of the CI method is to use a linear combination of $\mathrm{HF}$ wavefunctions in order to restore the various broken symmetries of the mean-field theory. In a doped system, for instance, the CI wavefunction is chosen to be a linear combination of $\mathrm{HF}$ wavefunctions describing the charged soliton centered at various sites. Besides restoring the translational symmetry, such a wavefunction also effectively takes into account the quantum dynamics of the charged soliton along the chain. This motion represents a large amplitude tunneling event between a given AFM mean field and the alternative AFM mean-field obtained by the operation $(S \rightarrow-S)$. Moreover, the propagating soliton lowers its quantum zero point energy considerably, relative to the static (HFA) soliton.

In Section 3 we briefly review the exact Bethe Ansatz (BA) solution and the ground-state energies of the halffilled and doped chains. The comparison between the Bethe Ansatz, Hartree-Fock and Configuration Interaction solutions is presented in Section 4. For both the undoped chain, and the chain with one doping hole, we show that through the nucleation of mobile quantum solitons, the CI method provides a much better description than the HFA. We identify the charge carrier of the Hubbard chain to be a charged bosonic domain-wall, and we show that its energy, in the CI method, is in excellent agreement with the BA result. The CI method leads to a simple, physical interpretation of spin-charge separation. Adding a single hole to a chain leads to the creation of two magnetic solitons. One soliton is the bosonic charged domain wall (which carries charge, but no spin), while the other soliton is the neutral spin-1/2 domain wall (which carries spin, but no charge). The energy of these excitations are in good agreement with the BA results. Finally, Section 5 contains discussion of the results and conclusions. 


\section{APPROXIMATIONS: HARTREE-FOCK AND CONFIGURATION INTERACTION METHOD}

\section{A. The Static Hartree-Fock Approximation}

One of the most widely used approximations for the many-electron problem is the Static Hartree-Fock Approximation (HFA). In this approximation the manybody problem is reduced to one-electron problems in which each electron moves in a self-consistent manner depending on the mean-field potential of the other electrons in the system. While this method is insufficient, by itself, to capture all of the physics of low dimensional electronic systems with strong correlations, it provides a valuable starting point from which essential fluctuation corrections can be included. In particular, we use the Hartree-Fock method to establish the electronic structure and the static energies of various magnetic soliton structures. In the more general Configuration Interaction (CI) variational wavefunction, the solitons acquire quantum dynamics and describe large amplitude tunneling and fluctuation effects that go beyond mean field theory.

In the $\mathrm{HF}$ approximation, the many-body wavefunction $|\Psi\rangle$ is decomposed into a Slater determinant of effective one-electron orbitals. The one-electron orbitals are found from the condition that the total energy of the system is minimized

$$
\delta \frac{\langle\Psi|\mathcal{H}| \Psi\rangle}{\langle\Psi \mid \Psi\rangle}=0
$$

In order to approximate the ground state of the Hamiltonian (1), we consider a Slater determinant trialwavefunction of the form

$$
|\Psi\rangle=\prod_{p=1}^{N_{e}} a_{p}^{\dagger}|0\rangle
$$

where $|0\rangle$ is the vacuum state, $N_{e}$ is the total number of electrons in the system and the one-electron states are given by

$$
a_{n}^{\dagger}=\sum_{i \sigma} \phi_{n}(i, \sigma) c_{i \sigma}^{\dagger}
$$

Here, the one-particle wave-functions $\phi_{n}(i, \sigma)$ form a complete and orthonormal system.

Using the wavefunction (3) in equation (2), and minimizing with respect to the one-particle wavefunctions $\phi_{n}(i, \sigma)$, we obtain the Hartree-Fock eigen-equations:

$$
\begin{gathered}
E_{n} \phi_{n}(i, \alpha)=-t \sum_{j \in V_{i}, \beta} T_{\alpha \beta}^{i j} \phi_{n}(j, \beta) \\
+U \sum_{\beta}\left(\frac{1}{2} \delta_{\alpha \beta} Q(i)-\vec{\sigma}_{\alpha \beta} \vec{S}(i)\right) \phi_{n}(i, \beta)
\end{gathered}
$$

where $\left(\sigma_{x}, \sigma_{y}, \sigma_{z}\right)$ are the Pauli spin matrices and the charge density,

$$
Q(i)=\left\langle\Psi\left|c_{i \alpha}^{\dagger} c_{i \alpha}\right| \Psi\right\rangle=\sum_{p=1}^{N_{e}}\left|\phi_{p}(i, \alpha)\right|^{2},
$$

and the spin density,

$$
\vec{S}(i)=\left\langle\Psi\left|c_{i \alpha}^{\dagger} \frac{\vec{\sigma}_{\alpha \beta}}{2} c_{i \beta}\right| \Psi\right\rangle=\sum_{p=1}^{N_{e}} \phi_{p}^{*}(i, \alpha) \frac{\vec{\sigma}_{\alpha \beta}}{2} \phi_{p}(i, \beta),
$$

must be computed self-consistently. The notation $j \in$ $V_{i}$ appearing in (5) means that the sum is performed over the sites $j$ which are nearest-neighbors of the site $i$. The self-consistent Hartree-Fock equations (5.6,67) must be satisfied by the occupied orbitals $p=1 \ldots N_{e}$, but can also be used to compute the empty (hole) orbitals.

The ground-state energy of the system in the HFA is given by

$$
E_{G S}=\langle\Psi|\mathcal{H}| \Psi\rangle=\sum_{p=1}^{N_{e}} E_{p}-U \sum_{i}\left(\frac{1}{4} Q(i)^{2}-\vec{S}(i)^{2}\right)
$$

where the single particle energies are obtained from (5).

The approximation scheme described so far is called the Unrestricted Hartree-Fock Approximation, because we did not impose constraints on the wavefunction $|\Psi\rangle$ which would require it to be an eigenfunction of various symmetry operations which commute with the Hamiltonian (1). If these symmetries are enforced, the method is called the Restricted Hartree-Fock Approximation. We use the Unrestricted HFA since it leads to lower energies. The breaking of symmetries in our case implies that electronig correlations are more effectively taken into account 12 The restoration of these symmetries is deferred until the CI wavefunction is introduced.

In the undoped (half-filled) case, the self-consistent Hartree-Fock equations can be solved analytically for the infinite system, using plane-wave one-particle wavefunctions. In the unrestricted Hartree-Fock approach, doping the system leads to the appearance of inhomogeneous solutions, which break the translational invariance. In this case, we solve the unrestricted self-consistent Hartree-Fock equations numerically on a finite chain. Starting with an initial spin and charge distribution $\vec{S}(i)$ and $Q(i)$, we numerically solve the eigenproblem (5) and find the $\mathrm{HF}$ eigenenergies $E_{n}$ and wavefunctions $\phi_{n}(i, \alpha)$. These are used in Eqs. (6) and (17) to calculate the new spin and charge distribution, and the procedure is repeated until self-consistency is reached. Numerically, we define self-consistency by the condition that the largest variation of any of the charge or spin components on any of the sites of the lattice is less that $10^{-9}$ between successive iterations. 


\section{B. Configuration Interaction Method}

The basic idea of the Configuration Interaction (CI) method is that the ground-state wavefunction, for a system with $N_{e}$ electrons, is not just a $N_{e} \times N_{e}$ Slater determinant (as in the HFA), but a judiciously chosen linear combination of such Slater determinants. 6 Given the fact that the set of all possible Slater determinants (with all possible occupation numbers) generated from a complete set of one-electron orbitals constitute a complete basis of the $N_{e}$-particle Hilbert space, our aim is to pick out a subset of Slater determinants which captures the essential physics of the exact solution.

Consider the CI ground-state wavefunction given by

$$
|\Psi\rangle=\sum_{i=1}^{N} \alpha_{i}\left|\Psi_{i}\right\rangle
$$

where each $\left|\Psi_{i}\right\rangle$ is a distinct $N_{e} \times N_{e}$ Slater determinant and the coefficients $\alpha_{i}$ are chosen to satisfy the minimization principle:

$$
\frac{\delta}{\delta \alpha_{i}}\left(\frac{\langle\Psi|\mathcal{H}| \Psi\rangle}{\langle\Psi \mid \Psi\rangle}\right)=0 \quad i=1, N
$$

This leads to the system of CI equations

$$
\sum_{j=1}^{N} \mathcal{H}_{i j} \alpha_{j}=E \sum_{j=1}^{N} \mathcal{O}_{i j} \alpha_{j} \quad i=1, N
$$

where $E=\langle\Psi|\mathcal{H}| \Psi\rangle /\langle\Psi \mid \Psi\rangle$ is the energy of the system in the $|\Psi\rangle$ state, $\mathcal{H}_{i j}=\left\langle\Psi_{i}|\mathcal{H}| \Psi_{j}\right\rangle$ are the matrix elements of the Hamiltonian in the basis of Slater determinants $\left\{\left|\Psi_{i}\right\rangle, i=1, N\right\}$, and $\mathcal{O}_{i j}=\left\langle\Psi_{i} \mid \Psi_{j}\right\rangle$ are the overlap matrix elements of the Slater determinants (which are not necessarily orthogonal). The CI solution is easily found by solving the linear system of equations (11), once the basis of Slater determinants $\left\{\left|\Psi_{i}\right\rangle, i=1, N\right\}$ is chosen. If we denote by $\phi_{p}^{(n)}(i, \sigma)$ the $p=1, \ldots, N_{e}$ one-electron occupied orbitals of the Slater determinant $\left|\Psi_{n}\right\rangle$, these matrix elements are given by:

$$
\mathcal{O}_{n m}=\left|\begin{array}{ccc}
\beta_{1,1}^{n m} & \ldots & \beta_{1, N_{e}}^{n m} \\
\vdots & & \vdots \\
\beta_{N_{e}, 1}^{n m} & \ldots & \beta_{N_{e}, N_{e}}^{n m}
\end{array}\right|
$$

The matrix elements of the Hamiltonian (11) can be written as:

$$
\mathcal{H}_{n m}=-t \cdot \mathcal{T}_{n m}+U \sum_{i} \mathcal{V}_{n m}(i)
$$

where the expectation values of the hopping and on-site interaction terms are:

$$
\mathcal{T}_{n m}=\sum_{p=1}^{N}\left|\begin{array}{ccccc}
\beta_{1,1}^{n m} & \ldots & t_{1, p}^{n m} & \ldots & \beta_{1, N_{e}}^{n m} \\
\vdots & & \vdots & & \vdots \\
\beta_{N_{e}, 1}^{n m} & \ldots & t_{N_{e}, p}^{n m} & \ldots & \beta_{N_{e}, N_{e}}^{n m}
\end{array}\right|
$$

and

$$
\mathcal{V}_{n m}(i)=\sum_{p_{1} \neq p_{2}}\left|\begin{array}{ccccccc}
\beta_{1,1}^{n m} & \ldots & u_{1, p_{1}}^{n m}(i) & \ldots & d_{1, p_{2}}^{n m}(i) & \ldots & \beta_{1, N_{e}}^{n m} \\
\vdots & & \vdots & & \vdots & & \vdots \\
\beta_{N_{e}, 1}^{n m} & \ldots & u_{N_{e}, p_{1}}^{n m}(i) & \ldots & d_{N_{e}, p_{2}}^{n m}(i) & \ldots & \beta_{N_{e}, N_{e}}^{n m}
\end{array}\right|
$$

Here,

$$
\begin{gathered}
\beta_{p h}^{n m}=\sum_{i \sigma} \phi_{h}^{(n) *}(i, \sigma) \phi_{p}^{(m)}(i, \sigma) \\
t_{p_{1}, p_{2}}^{n m}=\sum_{\substack{\langle i, j\rangle \\
\alpha \beta}}\left(\phi_{p_{1}}^{(n) *}(i, \alpha) T_{\alpha \beta}^{i j} \phi_{p_{2}}^{(m)}(j, \beta)+h . c .\right) \\
u_{p_{1}, p_{2}}^{n m}(i)=\phi_{p_{2}}^{(n) *}(i \uparrow) \phi_{p_{1}}^{(m)}(i \uparrow) \\
d_{p_{1}, p_{2}}^{n m}(i)=\phi_{p_{2}}^{(n) *}(i \downarrow) \phi_{p_{1}}^{(m)}(i \downarrow)
\end{gathered}
$$

We now consider the specific choice of the Slater determinant basis $\left\{\left|\Psi_{i}\right\rangle, i=1, N\right\}$. Strictly speaking, one may choose an optimized basis of Slater determinants from the general variational principle:

$$
\frac{\delta}{\delta \phi_{p}^{(n)}(i, \sigma)}\left(\frac{\langle\Psi|\mathcal{H}| \Psi\rangle}{\langle\Psi \mid \Psi\rangle}\right)=0 \quad n=1, N ; p=1, N_{e} .
$$

However, implementation of this full trial-function minimization scheme (also known as a multi-reference selfconsistent mean-field approach ${ }^{2}$ ) is numerically cumbersome even for medium-sized systems. Instead, we select the Slater determinant basis $\left\{\left|\Psi_{i}\right\rangle, i=1, N\right\}$ from the set of broken symmetry, Unrestricted Hartree-Fock wavefunctions (3), their symmetry related partners and their excitations. Clearly, (3) satisfies (14) by itself, provided that the $\alpha$ coefficients corresponding to the other Slater determinants in Eq. (9) are set to zero (see Eq. (2)). Since this Unrestricted HF wavefunction is not translationally invariant (the doping hole is always localized somewhere along the chain), we can restore the translational invariance of the CI ground-state wavefunction by also including in the basis of Slater determinants all the possible lattice translations of this Unrestricted HF wavefunction. In 2D, we must also include all the possible non-trivial rotations.

Clearly, all the translated HF Slater determinants lead to the same HF ground-state energy $\left\langle\Psi_{n}|\mathcal{H}| \Psi_{n}\right\rangle=E_{G S}$ as defined by Eq. (8). The CI method lifts the degeneracy between states with the hole-induced configuration localized at different sites, thereby restoring translational invariance. We may identify the lowering in the total energy due to the lifting of this degeneracy as quantum mechanical kinetic energy of deconfinement which the doping-induced configuration saves through hopping along the chain. In addition, quantum fluctuations in 
the internal structure of a magnetic soliton can be incorporated by including the lowest order excited state configurations of the static Hartree-Fock energy spectrum. Such wavefunctions are given by $a_{p}^{\dagger} a_{h}|\Psi\rangle$, where $p>N_{e}$ labels an excited particle state and $h \leq N_{e}$ labels the hole which is left behind (see Eq. (3)). Once again, all possible translations of this "excited" configurations must be included in the full CI wavefunction. These additions can describe changes in the "shape" of the soliton as it undergoes quantum mechanical motion through the crystal.

\section{EXACT SOLUTION OF THE 1D HUBBARD MODEL: THE BETHE ANSATZ}

Before reporting the results obtained in the $\mathrm{HF}$ and $\mathrm{CI}$ approximation for the 1D Hubbard model, we briefly de scribe the exact Bethe Ansatz solution of this problem, 2 for comparison purposes. Consider an $N$-site chain with $N_{e}$ electrons of which $M$ have spin-down. Here, $N_{e} \leq N$ and $M \leq N_{e} / 2$.2 Any wavefunction satisfying the manybody Schrödinger equation $\mathcal{H}|\Psi\rangle=E|\Psi\rangle$ is characterized by $N_{e}$ quasi-momenta $-\frac{\pi}{a}<k_{j} \leq \frac{\pi}{a}$ describing the motion of the $N_{e}$ electrons ( $a$ is the lattice constant), and $M$ rapidities, $\Lambda_{\alpha}$ describing the spin ordering. Using the Bethe AnsatzB (BA) and imposing periodic boundary conditions, it can be shown that the quasimomenta and therapidities satisfy the so-called Bethe Ansatz equations 213

$$
\begin{gathered}
\exp \left(i k_{j} N a\right)=\prod_{\alpha=1}^{M} \frac{\sin \left(k_{j} a\right)-\Lambda_{\alpha}+\frac{U}{4} i}{\sin \left(k_{j} a\right)-\Lambda_{\alpha}-\frac{U}{4} i} \\
\prod_{j=1}^{N_{e}} \frac{\Lambda_{\alpha}-\sin \left(k_{j} a\right)+\frac{U}{4} i}{\Lambda_{\alpha}-\sin \left(k_{j} a\right)-\frac{U}{4} i}=-\prod_{\beta=1}^{M} \frac{\Lambda_{\alpha}-\Lambda_{\beta}+\frac{U}{2} i}{\Lambda_{\alpha}-\Lambda_{\beta}-\frac{U}{2} i}
\end{gathered}
$$

The total energy and the total crystal momentum are then given by

$$
E=-2 \sum_{j=1}^{N_{e}} \cos \left(k_{j} a\right) \quad P=\sum_{j=1}^{N_{e}} k_{j}
$$

The ground state is always given by real $k$ 's and $\Lambda$ 's. Excited states are usually described by complex rapidities in so-called "string" structures.

We solve the Bethe Ansatz equations iteratively, starting with a guess for the set of real rapidities $\Lambda_{\alpha}$ (related to the ground-state solution of the 1D Heisenberg chain, as described in Reference13). Then, we solve Eq.(15) and find the quasi-momenta $k_{j}$, which we use in Eq.(16) to find the new set of real rapidities. The procedure is repeated until self-consistency is reached.

We can check our numerical procedure in two particular cases. First, the ground-state energy of a half-filled
$N=N_{e}$ chain in the thermodynamic limit is known to be given by 212

$$
E=-4|t| N \int_{0}^{\infty} \frac{d x J_{0}(x) J_{1}(x)}{x(1+\exp (x U /(2|t|)))}
$$

where the $J_{\nu}(x)$ are cylindrical Bessel functions. In Fig. 11 we plot the ground-state energy per site, in units of $t$, obtained for a half-filled chain of various lengths $N$, for $U / t=5$. While for very low values of $N$ there are large variations between the energies of chains with even and odd number of unit cells, as $N$ increases the energies obtained converge towards the thermodynamic value of Eq. (18) (shown as the full line). We consider chains with an even number of sites (integer number of unit cells), since we know that the ground-state has AFM correlations. Similar curves are obtained for other values of $U / t$.

Another well known case is that of a chain with just one hole, in the $U / t \rightarrow \infty$ limit. In this limit, double occupancy is forbidden by the large on-site interaction, and the only possible charge fluctuation is the motion of the hole. The total energy of the chain reduces to the energy of the hole, since the single occupied sites give little contribution to energy (in this limit the contributions from the AFM correlations of the electron spins, of the order of $t^{2} / U$, are negligible for finite chains). It is straightforward to show 14 that the hole's dispersion relation in $1 \mathrm{D}$ is exactly that of a free particle, and therefore the total energy of the chain containing a single hole with momentum $\hbar k$ is $E(k)=-2 t \cos (k a)$. In the following sections, we show that the ground-state energy approaches $E=-2 t$ as $U / t \rightarrow \infty$ for the CI method as well as for the Bethe ansatz equations of the chain with one hole.

\section{COMPARISON BETWEEN THE BA, THE CI AND THE HF RESULTS}

\section{A. The undoped ground-state}

\section{Hartree-Fock results}

For the undoped system, the self-consistent HF equations $(5,6,6)$ for an infinite system give rise to a meanfield ground state with long range antiferromagnetic order. However, the Mermin-Wagner theorem states that the true ground-state of any one dimensional isolated system cannot have long range order (LRO) and that LRO is absent in 2D systems for non-zero temperatures. In the framework of the CI method, mobile solitons in the AFM background mediate the destruction of LRO.

Using the spin and charge distributions $Q(i)=1$ (one electron per site) and $\vec{S}(i)=(-1)^{i} S \vec{e}_{z}$ (AFM order in the arbitrary direction $\vec{e}_{z}$ ), equation (5) yields two electronic bands characterized by the dispersion relations

$$
E_{k \sigma}^{c / v}= \pm \sqrt{\epsilon_{k}^{2}+(U S)^{2}} \quad k \in(-\pi / 2 a, \pi / 2 a]
$$


where $\epsilon_{k}=-2 t \cos (k a)$ is the dispersion relation of noninteracting electrons and $a$ is the lattice constant. Each of these levels is double-degenerate $(\sigma= \pm 1)$. Given the symmetry of the spectrum and the fact that only half the states are occupied, one can easily see that in the Hartree-Fock ground state, all the states in the valence band $\left(E_{k}^{v}<0\right)$ are occupied, while all the states in the conduction band $\left(E_{k}^{c}>0\right)$ are empty. The two bands are separated by the usual Mott-Hubbard charge transfer gap opened at the Fermi surface $(k= \pm \pi / 2 a)$, of magnitude $2 U S$.

Using the valence band wavefunctions in Eqs. (6) and (7), we obtain 15 the self-consistent spin amplitude

$$
S=\frac{U S}{N} \sum_{k} \frac{1}{\sqrt{\epsilon_{k}^{2}+(U S)^{2}}}
$$

where $N$ is the number of sites and the sum is performed over the Brillouin zone $k \in(-\pi / 2 a, \pi / 2 a]$. This equation has three solutions. One is trivial $(S=0)$. For $S \neq 0$ the equation depends only on $S^{2}$. Consequently, the meanfield ground state is doubly-degenerate: both $+S$ and $-S$ satisfy Eq. (20) and give rise to self-consistent groundstates which differ from each other only through the fact that all the spins are flipped from one ground-state to the other one. Strictly speaking, the HF solution gives an infinite number of degenerate ground states, because the direction $\vec{e}_{z}$ is arbitrary (this is a direct consequence of the fact that the Hubbard model is rotationally invariant). However, since a real chain is embedded in a 3D crystal, crystal field interactions will lift the rotational degeneracy, and fix one particular direction of orientation (easy axis) for the spins (for instance, along the chains). Consequently one particular direction $\vec{e}_{z}$ is favored, and the mean-field ground state is doubly degenerate.

Since all the states of the valence band are occupied, the energy of the HF ground state is simply given by:

$$
E_{G S}=2 \sum_{k} E_{k}^{v}+N U\left(S^{2}+\frac{1}{4}\right)
$$

where $S$ is given by the self-consistency condition 20. The magnitude of the ground state energy per site, in units of $t$, is plotted in Fig. (2) as a function of $U / t$ (the full line). The following features can be observed: in the $U / t \rightarrow 0$ limit (noninteracting electrons), the energy of the ground state has, indeed, the expected value $E_{G S} \rightarrow$ $-4 N t / \pi$. In the strong interaction limit $U / t \rightarrow \infty$, the energy of the ground state goes to zero as expected (since in this limit every site is single occupied and virtual hopping is suppressed). For comparison, the exact groundstate energy obtained from the Bethe Ansatz (Eq. (18)) is also shown (dashed line). The asymptotic value of the $\mathrm{HF}$ energy is found to be given by $E_{G S} \rightarrow-2 N t^{2} / U$. It is well known that in this limit, the Hubhard model is equivalent to an AFM Heisenberg model 16 with a coupling constant $J=4 t^{2} / U$, and that its true ground state energy per site is $E_{G S} \rightarrow-N J \ln 2=-2.77 N t^{2} / U$.
This suggests that the Hartree-Fock method is a good starting point, from which to incorporate fluctuation corrections which lower the energy.

\section{Configuration Interaction treatment of the undoped chain}

The HF Slater determinant of the undoped AFM ground state is invariant to translations by $2 a$ (AFM order must be preserved). While it is possible to include in the CI set of Slater determinants excited HF states of the undoped chain obtained by exciting electrons from the valence to the conduction band, it turns out that lower energy self-consistent HF configurations can be generated by breaking the translational symmetry of the undoped mean-field AFM background. This is facilitated by considering excited states of the AFM background which can accommodate charge carriers in localized states deep within the charge transfer gap rather than within the Mott-Hubbard bands. The lowest energy self-consistent excited state is the undoped (neutral) domain-wall, which describes tunneling from one mean-field ground-state to the other mean-field ground-state. Since the AFM order rotates by $\pi$ when crossing the domain wall, we consider either one domain wall on an odd-site chain, or a pair of domain walls on an even-site chain, in order to impose cyclic boundary conditions. Fig. 3 depicts a typical selfconsistent configuration containing two neutral domain walls, one centered between sites 10 and 11 , and one centered between sites 30 and 31 . The charge $Q(i)=1 \mathrm{ev}$ erywhere. Near the domain wall the self-consistent spin magnitudes $S_{z}(i)$ adjust such that each neutral domain wall carries a spin $1 / 2$ (with a projection in the same direction as its core spins), suggesting that this excitation is a neutral fermion. This is confirmed from the electronic structure shown in Fig.4.

We obtain self-consistent configurations containing two neutral domain-walls at all possible distances from each other, either having opposite orientations (i.e. total chain spin 0 ) or same orientations (total chain spin \pm 1 ). Since the AFM ground state has total spin 0 and states with different total spin do not mix, we need only include in the set of CI Slater determinants $\left|\Psi_{i}\right\rangle$ states of total spin zero, i.e. those having the neutral domain walls "paired" (with opposite orientations). Since all the possible configurations with two neutral domain-walls have very similar energies, they must all be included along with the AFM undoped ground state in making up the variational trial wavefunction. We must also include both AFM undoped mean-field ground-states in the CI set. This can be easily seen from Fig.3, where half of the chain is in one AFM ground state, and the other half is in the other (flipped) ground state. Therefore, this state will have equal overlap with both AFM ground states, although the AFM ground-states themselves are orthogonal to each other. For an $N$-site chain, the CI set contains a total of $2+\mathrm{N}(\mathrm{N}-$ 2)/2 Slater determinants, two being the undoped AFM 
ground states, and the rest being the N-2 possible states with paired domain-walls at different distances from each other, each of which can be translated N/2 times along the chain.

The total ground-state energy found with the $\mathrm{HF}$ (circles), CI (squares) and BA (diamonds) methods for chains of different lengths $N$ for $U / t=5$ and $U / t=50$ are shown in Fig.5. For all three methods the total energy of the chain is proportional to the length of the chain. The addition of the configurations with a pair of neutral domain walls in the CI method improves the ground-state energy considerably. It is obvious, however, that as the length of the chain increases configurations with two, three, four and more pairs of neutral domain walls should be included in the CI set in order to arrive at a perfect agreement with the exact Bethe Ansatz solution. It is interesting to remark that even if only the configurations with one pair of neutral domain walls are included, the non-degenerate CI ground state is such that $\left\langle S_{z}(i)\right\rangle=0$ for any site $i$ of the chain, although the antiferromagnetic correlations remain very strong. This is a consequence of the fact that the CI set of Slater determinants contains an equal number of states with the spin at the site $i$ up and down, so in average each spin expectation value is vanishing. Thus, the CI wavefunction is much more successful in mimicking the properties of the exact BA ground-state.

\section{B. Charged solitons in the doped ground state: the spin-bag and the charged domain-wall}

\section{Hartree-Fock results}

If we numerically solve the HF equations (5,6, 6,7) for a $N$-site chain with $N-1$ electrons, we find three types of charged self-consistent solutions: the spin-bag (or spinpolaron) (Fig.6), the charged domain-wall centered on site (COS domain wall) (Fig.7) and the charged domainwall centered between sites (CBS domain wall) (Fig.8). The spin-polaron is created by trapping the hole in a small ferromagnetic core, which only affects the LR AFM order locally. The domain walls are topological excitations, since the AFM order is rotated by $\pi$ as one goes through the domain wall. Therefore, in order to impose cyclic boundary conditions, we must consider an oddsite chain (or we may take an even-site chain and add two holes, leading to the appearance of a domain wallanti-domain wall pair). The localization length of the hole decreases as $U / t$ increasing for all three excitations. The spin and charge at sites far from the distortion equal the undoped mean-field ground-state values.

The electronic spectra corresponding to the configurations shown in Figs. 6., and 8 are shown in Figs. 9 and 10. First panel in Fig. 9 corresponds to the undoped ground state of a $N=40$ sites chain. As discussed before, the electronic spectrum consists of two bands of $N=40$ states each. The valence band is completely filled, the conduction band is completely empty, and there is a large charge transfer gap between them. Adding one hole on the same $N=40$ sites chain, and keeping the cyclic boundary conditions, leads to the appearance of the spin-bag shown in Fig.6. Its electronic structure is shown in the right panel of Fig.9. There is a localized level $(n=1)$ well below the valence band, the valence band contains 38 states, there are 3 localized levels deep inside the Mott-Hubbard charge transfer gap, and finally the conduction band also has 38 levels. Since there are $N-1=39$ electrons in the system, only the localized level below the valence band and the valence band states are occupied. Since the valence band is spin paired (having an even number of states), this means that the total spin of this excitation is $1 / 2$, associated with the spin of the electron on the localized level. The fact that the spin-bag carries a $1 / 2$-spin is also easy to deduce from Fig. 6, because of the small ferromagnetic core. Thus, we conclude that the spin-bag is a charged fermion.

The charged domain wall electronic structures are shown in Fig. 10, with the CBS domain wall in the left panel, and the COS domain wall in the right panel. In this case, we study a chain with $N-1=40$ electrons and $N=41$ sites, so that we can impose cyclic boundary conditions again. We can see that in both cases there are 4 localized levels inside the Mott-Hubbard gap, $N-1=40$ occupied states in the valence band, and 38 states in the conduction band. The localized levels of the COS domain wall are degenerate. The degeneracy is lifted for the CBS domain wall, and the upper discrete level is pushed quite close to the lower edge of the conduction band. However, it is still a localized level (this is easily checked by plotting its wavefunction). In both cases we have a fully spin-paired valence band, and therefore the total spin of these excitations is zero. Since they carry the charge of the hole, the domain walls are charged bosqn, in analogy to the charged solitons of polyacetylene.17.18

In order to establish the relevance of these different charged spin configurations, we compare their excitation energies (defined with respect to the undoped (half-filled) ground state) as a function of $U / t$ in Fig. (11). As we can see, the domain walls are the low-energy excitations for $U / t<6.5$, while the spin-polarons become the lowenergy excitations for $U / t>6.5$. As $U / t \rightarrow 0$, the core size of the domain-walls diverges roughly like $t / U$. As a result, in this limit the COS and the CBS domain-walls are very extended objects which become indistinguishable and degenerate. However, as $U / t$ increases the core becomes more and more localized, and the CBS domainwall becomes energetically favorable relative to the COS domain-wall.

However, this static HFA does not take into account the lowering of energy of these excitations due to translations along the chain. From the simple inspection of the spin distributions of the spin-bag and of the domain wall, we can easily deduce that while a domain wall can 
move freely along the chain, the spin-bag is rather immobile. Moving the center of the domain wall by one site (by interchanging the hole with the spin at the right or at the left) necessitates only some rearrangement of the magnitude of the core spins, while their orientation is automatically correct. Consequently, the domain wall lowers its energy by an amount of order $t$ through hopping along the chain. However, if a spin-bag moves only by one site, the translated spin must be flipped (which would require the the total spin of the spin-bag to likewise flip). In order to conserve its spin, the spin-bag must tunnel to the second nearest neighbor. This is a second order process, and consequently the spin-bag lowers its energy only by an amount of order $t^{2} / U$ through motion. As we demonstrate below, using the Configuration Interaction method, these qualitative arguments are valid. When soliton dynamics is incorporated, it is the charged bosonic domain wall that proves to be the relevant charged excitation of the Hubbard model for all values of $U / t$.

\section{Configuration Interaction treatment of the spin-polaron}

Consider a spin-polaron on a chain with $2 N$ sites (the number of sites is even so that we can impose cyclic boundary conditions). Using the CI method we evaluate the kinetic energy of the mobile, charged spin-bag. As suggested above, we only need to include in the set of Slater determinants $\left|\Psi_{i}\right\rangle$ configurations translated by an even number of sites from the initial HF configuration. Let $\left|\Psi_{\text {even }}\right\rangle,\left|\Psi_{\text {odd }}\right\rangle$ be the HF determinants for the spinpolaron centered at an even and odd site, respectively, and let $\hat{S}_{z}=\sum_{i} \hat{S}_{z}(i)=\frac{1}{2} \sum_{i, \sigma} \sigma c_{i \sigma}^{\dagger} c_{i \sigma}$ be the total spin operator in the $z$-direction. Then, $\hat{S}_{z}\left|\Psi_{\text {even }}\right\rangle=\frac{1}{2}\left|\Psi_{\text {even }}\right\rangle$ while $\hat{S}_{z}\left|\Psi_{\text {odd }}\right\rangle=-\frac{1}{2}\left|\Psi_{\text {odd }}\right\rangle$ (or viceversa), and therefore $\left\langle\Psi_{\text {odd }} \mid \Psi_{\text {even }}\right\rangle=0$. Since the Hubbard Hamiltonian commutes with $\hat{S}_{z}$, it follows that $\left\langle\Psi_{\text {odd }}|\mathcal{H}| \Psi_{\text {even }}\right\rangle=0$. From the CI equation (11) we conclude that there is no mixing between states with the spin-polaron on one sublattice and states with the (opposite spin) spin-polaron on the other sublattice. Therefore, on a chain with $2 N$ sites we only need to mix $N$ Slater determinants in order to obtain the spin-polaron ground-state within the CI method.

If the initial self-consistent $\mathrm{HF}$ spin-polaron configuration $\left|\Psi_{1}\right\rangle$ is composed of the one-particle occupied orbitals $\phi_{p}^{(1)}(i, \sigma)$, the one-particle orbitals of the state $\left|\Psi_{n+1}\right\rangle$ translated by $2 n a$ will simply be chosen as $\phi_{p}^{(n+1)}(i, \sigma)=\phi_{p}^{(1)}(i-2 n, \sigma)$ (cyclic boundary conditions are assumed). The overlap matrices $\mathcal{O}_{n m}$ and $\mathcal{H}_{n m}$ are then calculated and the CI matrix equation (11) solved. Numerically, the largest amount of time is spent computing the $\mathcal{H}_{n m}$ matrix elements. Due to various symmetries, there are only $N / 2$ distinct matrix elements.

Given the structure of the CI equation (11), we can readily see that its solutions are of the form

$$
\left|\Psi_{k}\right\rangle=\sum_{n=1}^{N} e^{(i k n 2 a)}\left|\Psi_{n}\right\rangle
$$

where $a$ is the lattice constant and there are $N$ distinct $k$ values. These values satisfy the periodicity condition $\exp (i k N 2 a)=1$ since translating any spin-polaron configuration by the total chain length $2 \mathrm{Na}$ leaves the configuration unchanged. Therefore, the distinct wavevectors are $k=m \pi / N a, m=0,1, \ldots, N-1$. The reduced Brillouin zone $[0, \pi / a)$ (or, symmetrically, the $(-\pi / 2 a, \pi / 2 a]$ interval) is due to the motion of the spin-polaron on only one sublattice, and corresponds to states with spinup (for instance). The band corresponding to the spinpolaron on the other sublattice will have the same structure, but corresponds to spin-down states. In the end we recover the typical spin-degenerate band expected for fermions.

Given the general form of the wavefunction, the dispersion relation of the spin-polaron follows from the expression

$$
E(k)=\frac{\left\langle\Psi_{k}|\mathcal{H}| \Psi_{k}\right\rangle}{\left\langle\Psi_{k} \mid \Psi_{k}\right\rangle}=\frac{\sum_{n=1}^{N} \exp (2 i k a(n-1)) \mathcal{H}_{1 n}}{\sum_{n=1}^{N} \exp (2 i k a(n-1)) \mathcal{O}_{1 n}}
$$

In deriving the last equation, we used the symmetry properties of the matrices $\mathcal{H}_{n m}$ and $\mathcal{O}_{n m}$, namely that the $(\mathrm{nm})$ matrix element only depends on $n-m$. Strictly speaking, $E(k)$ is the energy of the whole chain containing the spin-polaron and will strongly depend on the length of the chain. We extract the dispersion relation of the spin-polaron from a fit of the form

$$
E(k)=2 N e_{G S}+E_{p o l}(k)
$$

Here $e_{G S}$ is interpreted as the ground-state energy per site of the undoped AFM (for a very long chain, most of the sites are unaffected by the presence of the single spinpolaron). We define $E_{p o l}(k)$ as the dispersion relation of the spin-polaron itself. In other words, the energy of the spin-polaron is defined as the difference between the energy of the chain with the spin-polaron, and that of an undoped chain.

We plot $E_{\text {pol }}(k)$ versus $k$ in Fig. 12, for $U / t=5$ and chains of various lengths. The various curves fall on top of each other, thus proving that the fit (24) is legitimate. Also shown is the excitation energy of the static holedoped spin-polaron $E_{\text {pol }}^{H F}$ (the full line), as obtained from the Unrestricted HFA (also defined with respect to the energy of the undoped chain). Clearly, translation lowers the total energy of the spin-polaron, with the most stable state corresponding to $k=\pi / 2 a$. The total kinetic energy gained is, however, only of the order of $t^{2} / U$. This is shown in Fig. 13, where we plot both the kinetic energy gained $E_{\text {pol }}\left(\frac{\pi}{2 a}\right)-E_{\text {pol }}^{H F}$ (circles) and the width of the spin-polaron band, $E_{\text {pol }}\left(\frac{\pi}{2 a}\right)-E_{\text {pol }}(0)$ (squares), as a function of $t^{2} / U$. The log-log graph is linear with a slope of unity as expected, since the spin-polaron must tunnel two sites (second order hopping process) to the next 
spin-allowed position. Clearly, this charged fermionic excitation is relatively immobile.

We conclude that in the large $U / t$ limit the CI correction to the spin-bag energy is negligible, due to the immobility of this excitation. As a result, the energy of the spin-bag varies with $U / t$ as shown in Fig.11, for large $U / t$, and it saturates above $-1.5 t$ as $U / t \rightarrow \infty$. As already discussed, it is known that in the $U / t \rightarrow \infty$ limit the energy of the doping hole is $-2 t$. This discrepancy suggests that the spin-bag does not provide a good description for the charge carrier.

\section{Configuration Interaction treatment of the charged domain-wall}

To investigate an isolated charged domain wall, we consider chains with an odd number $(2 N+1)$ of sites. As shown in Figs. 7 and 8, there are two types of selfconsistent charged domain-walls, namely the COS (centered on site) domain wall and the CBS (centered between sites) domain wall. Since the charged domain walls are bosons $\left(S_{z}|\Psi\rangle=0\right)$, there is non-vanishing overlap between states with the domain-wall centered on different sublattices. Unlike the fermionic spin-polarons, we must include all possible translations in the CI Slater determinant set $\left|\Psi_{i}\right\rangle$. For a $2 N+1$-site chain, there are $2(2 N+1)$ distinct COS domain wall configurations, and $2(2 N+1)$ distinct $\mathrm{CBS}$ domain wall configurations. The reason for the factor 2 is that translation of a domain wall by $2 N+1$ sites takes it into a domain wall centered at the same site as in the initial configuration, but with all spins flipped, due to the $\pi$-difference in the AFM ordering of the spins on the two sides of the domain wall. A second translation around the whole chain is necessary in order to regain the initial configuration. As a result, there is a four-fold increase in the number of possible configurations for a domain wall, as compared to a spin-polaron on a chain of almost the same length. We generate the translations with an even number $2 n$ of lattice sites in the same way as for the spin-polaron, $\phi_{p}^{(2 n+1)}(i, \sigma)=\phi_{p}^{(1)}(i-2 n, \sigma)$ if $0 \leq n \leq N$ (first translation around the chain) and $\phi_{p}^{(2 n+1)}(i, \sigma)=\phi_{p}^{(1)}(i-2 n,-\sigma)$ if $N<n \leq 2 N$ (second translation around the chain). Here, we remind the reader that $\phi_{p}^{(m)}(i, \sigma)$ represents a particular $(p)$ occupied one-electron orbital of the static Hartree-Fock Slater determinant $\left|\Psi_{m}\right\rangle$ which places a static magnetic soliton at site $m$. For translations with an odd number $2 n-1$ of sites, the wavefunction mapping is $\phi_{p}^{(2 n)}(i, \sigma)=$ $\phi_{p}^{(1)}(i-(2 n-1),-\sigma)$ if $1 \leq n \leq N$ (first translation around the chain) and $\phi_{p}^{(2 n)}(i, \sigma)=\phi_{p}^{(1)}(i-(2 n-1), \sigma)$ if $N<n \leq 2 N+1$ (second translation around the chain).

Let us first consider only including one type of domain wall (either COS, or CBS), in the CI wavefunction. In this case, we can again conclude that the solutions of Eq. (11) must be of the form

$$
\left|\Psi_{k}\right\rangle=\sum_{n=1}^{2(2 N+1)} e^{i k n a}\left|\Psi_{n}\right\rangle
$$

where $\left|\Psi_{n}\right\rangle$ is the configuration translated by $n-1$ sites from the initial HF configuration $\left|\Psi_{1}\right\rangle$. The periodicity condition is now $e^{i k 2(2 N+1) a}=1$ and the allowed values of $k$ are given by $k=m \pi /(2 N+1) a, m=$ $0,1, \ldots, 2(2 N-1)-1$. Clearly, the domain-wall dispersion band is extended over the full Brillouin zone $[0,2 \pi$ ) (or the symmetric version $(-\pi, \pi])$. The dispersion relation is given by

$$
E(k)=\frac{\left\langle\Psi_{k}|\mathcal{H}| \Psi_{k}\right\rangle}{\left\langle\Psi_{k} \mid \Psi_{k}\right\rangle}=\frac{\sum_{n=1}^{2(2 N+1)} \exp (i k(n-1) a) \mathcal{H}_{n 1}}{\sum_{n=1}^{2(2 N+1)} \exp (i k(n-1) a) \mathcal{O}_{n 1}}
$$

As in the case of the spin-polaron, we extract the dispersion relation of the domain-wall from $E(k)$ by subtracting the energy of the undoped chain

$$
E_{d w}(k)=E(k)-(2 N+1) e_{G S}
$$

In Fig. 14 we show the dispersion relations $E_{d w}(k)$ vs. $k$ for both CBS (left panel) and COS (right panel) domain walls on chains of different length $2 N+1$, and $U / t=5$. The excitation energy of the static configuration (obtained from the unrestricted HF search) is also shown. Again, various dispersion curves fall on top of each other, validating the fit of Eq. (27). Comparing Fig. 14 with Fig. 12, it is immediately apparent that the dispersion band of the domain walls is much wider. In fact, the band of the COS domain wall extends up to $4 t$ (not shown). Comparing the bottom of the dispersion band to the static HF excitation energy of domain wall (shown as a full line), we see that translational motion lowers the energy of the domain wall by about $t$ (as opposed to only $0.3 t$ when $U / t=5$ for a spin-polaron). While the bottom of the dispersion band is basically identical for both types of domain walls, the top is very different. Excited states with energy $E(k)>0$ require the incorporation of the excited state configurations of the single Slater determinant (from static Hartree-Fock) in the CI set. Clearly these excited state, static configurations have energies comparable to the moving domain wall at high energy parts of the dispersion curve. The most likely candidates are those configurations in which electrons from the top of the valence band are excited into the bound discrete levels. If only one such excitation takes place, the energy of the static configuration is raised by $\approx U / 2$ (the difference between the energy of the level at the top of the valence band, and that of the first empty localized level). By mixing such configurations in the CI Slater determinant set, we obtain modifications to the upper part of the dispersion relation, while the bottom remains unchanged. Since we are interested in the kinetic energy gained by the domain wall through translation $(E(k)<0$ region), we will neglect these higher-energy processes in what follows. 
A technical issue that emerges is the effect of mixing both the COS and the CBS domain wall configurations when calculating the CI wavefunction. While one might hope for an improvement in the overall energy for the mobile, charged soliton, this is not the case. The reason is that each set of configurations by itself generates basically the same CI wavefunctions $\left|\Psi_{k}\right\rangle$ at the bottom of the dispersion band rather than linearly independent ones. This can easily be seen numerically if we analyze the eigenvalues of the overlap matrix $\mathcal{O}_{n m}$. Suppose that $\lambda$ is an eigenvalue of this matrix, and that $\left(\alpha_{i}\right)_{i=1, N}$ is the corresponding eigenvector (for simplicity, we use $N$ as the dimension of the overlap matrix). Defining $|\Psi\rangle=\sum_{i=1}^{N} \alpha_{i}\left|\Psi_{i}\right\rangle$ it is straightforward to show that $\langle\Psi \mid \Psi\rangle=\lambda$. When we mix both sets of configurations together, we find many vanishing eigenvalues $\lambda=0$, which imply $|\Psi\rangle=0$ (numerically, we use the Singular Value Decomposition technique as a diagnostic for vanishing eigenvalues). This proves that there are redundant linearly dependent combinations in the set of Slater determinants $\left|\Psi_{i}\right\rangle$. We remove these linearly dependent states to find the CI ground-state. In particular, by mixing COS and CBS domain wall configurations, the resulting low-energy spectrum is the same as that found by using only the lower energy CBS domain wall configurations.

The previous analysis gives us the lowest energy of a single hole (charged domain wall soliton) on the chain that can be obtained within the CI approximation. In comparing this energy to the one obtained from the exact Bethe Ansatz solution, it is not appropriate to directly compare the total chain energies. The reason, as already proved, is that there is a large contribution to these energies proportional to the number of sites in the chain, the proportionality constant being the undoped ground-state energy per site (see Eq. (27)). The HFA gives a higher undoped energy per site than the exact BA energy (see Fig. 2), and the CI approach does not improve it unless we also add states with pairs of uncharged domain-walls. Our aim is to isolate the energy of the doping hole. Therefore, we compare $E_{d w}\left(\frac{\pi}{a}\right)$ (the lowest CI energy of the domain wall itself) with the corresponding doping hole energy extracted from the Bethe Ansatz. This comparison is shown in Fig. 15. In order to find the doping hole energy from the Bethe Ansatz, we evaluate the exact ground-state energy of a chain with $2 N+1$ sites and $2 N$ electrons ( half $M=N$ of which have spin-down), for various values of $N$. This set of energies is seen to be well fitted by an expression of the form $E(N)=(2 N+1) e_{G S}+E_{0}$, where $e_{G S}$ is in excellent agreement with the BA ground-state value predicted by (18). As in the CI approach, we identify $E_{0}$ with the energy of the hole. The BA energies of the hole, as a function of $U / t$ are shown as squares in Fig. 15. They indeed go to $-2 t$ in the $U / t \rightarrow \infty$ limit, as expected. In the $U / t \rightarrow 0$ limit, the energy of the doping hole is expected to go to zero, since in this $U=0$ limit the system is a metal.
The CI domain-wall energies $E_{d w}=E_{d w}\left(\frac{\pi}{a}\right)$ are shown as full circles in Figure 15. The agreement with the Bethe Ansatz energy is striking. For $U / t \geq 5$, the fit (27) is excellent and the error bars on the domain wall energies are extremely small. However, as $U / t \rightarrow 0$, the size of the domain wall increases significantly (it is around 20 sites for $U / t=2$ ) and therefore extremely long chains need to be considered for a good fit. The two upper lines correspond to the static HF energies obtained for a selfconsistent CBS domain wall (triangle down) and a COS domain wall (triangle up). The diamonds show the CI results for the spin-polaron. Clearly, the translational motion of the domain wall (included in the CI approach) drastically lowers its overall energy. The kinetic energy saved is of the order $t$ over most of the $U / t$ parameter range.

The agreement between the domain-wall energy as calculated in the CI approach and the exact doping-hole energy as given by the Bethe Ansatz is quite remarkable, over the whole range of $U / t$ parameters. The only disagreement appears for $U / t \leq 2$, where the domainwalls become extremely delocalized and the numerical calculations are very difficult. The CI solution is not exact because the HF description neglects the presence of additional neutral domain-walls pairs in the AFM background. While the addition of such pairs improves the accuracy of the CI method relative to the exact solution, it makes the calculation more cumbersome, due to the large increase in the number of possible configurations. When the contribution of this background is removed, the CI solution of a single charged domain wall moving around the chain agrees very well with the exact solution. This suggests that the renormalization of the charged domain wall energy by neutral domain wall pairs is relatively small. The agreement with the Bethe Ansatz is not limited to the bottom of the charged domain wall dispersion curve. In the large $U / t$ limit, the domain-wall dispersion band is indeed given by $E_{d w}(k)=2 t \cos (k a)$, as required.14 For example, the dispersion relation for domain-wall corresponding to $U / t=100$ is shown in Fig. 16. At such high $U / t$ values, the typical energy of the configurations containing electrons excited on the midgap levels is of order $U / 2$. They do not influence the lowest domain wall band. As $U / t$ is decreased, these excited configurations simply modify the high energy part of the domain-wall dispersion relation.

In conclusion, the mobile charged bosonic domain wall excitation is the relevant charged excitation of the $1 \mathrm{D}$ chain described by the Hubbard model for all values $U / t$. Although the static charged spin-polaron has lower excitation energy than the static domain-wall for $U / t>6.5$, when quantum dynamics is taken into consideration (CI method) the mobile charged domain wall turns out to be the lowest energy excitation. 


\section{Spin-charge separation}

A particularly striking effect can be recaptured in the CI method if one adds a hole to an even-site chain. In the HFA, this leads to the appearance of one spin-polaron, since a single charged domain wall is incompatible with the cyclic boundary conditions involving an even number of sites. In the CI method, this charged spin-polaron is unstable to dissociation into a pair of more mobile domain-wall solitons. In particular the charged spin-bag dissociates into a charged bosonic domain-wall and a neutral fermionic domain wall. The translational kinetic energy saved by the domain wall motion more than offsets the additional exchange energy cost in creating a pair of solitons from a single spin-polaron.

We demonstrate this spin-charge separation effect using the set of all the possible configurations with a pair of a charged and a neutral domain walls in the CI method. Again, since states with different spins do not mix, we need only keep configurations in which the uncharged domain wall has the same spin, either $+1 / 2$, or $-1 / 2$. As in the undoped case, configurations with a pair of domain walls connect the two possible AFM ground states. This leads to a considerable change in the background energy (see Fig.5). As a result, we define the energy of the doping hole, in this case, with respect to the CI energy of an undoped chain with a pair of neutral domain-walls. This allows us to properly account for the renormalization of the background energy of the chain by the pair of domain walls. Physically, we interpret this in the following way. The true ground state of the undoped chain has a certain number of pairs of neutral domain walls. When the chain is doped, the doping hole is bound into one of the already existing neutral domain walls, transforming it into a charged bosonic domain wall. All the other pairs of neutral domain walls remain largely unaffected.

The results of this analysis are plotted in Fig.17. We use the Bethe equations to calculate the ground-state energy of even-site chains with one doping hole. The BA excitation energy for the doping hole added to an evensite chain is shown as circles. The exact chain energy is found to be well fitted by $E(N)=N e_{G S}+E_{o}$, with $N$ being the even number of sites of the chain, and $e_{G S}$ the undoped ground-state energy per site. Since $N e_{G S}$ is the total energy of the undoped chain, we again identify $E_{o}$ as the energy of the doping hole. The energy of a charged domain wall - neutral domain wall pair, obtained using the CI method for even-site chains, is shown as squares. Clearly, the CI method recaptures the physics as well as the energetics of the BA solution to a high degree of accuracy. Even closer agreement can be achieved if configurations with more pairs of uncharged domain-walls are included in the CI basis set. This result provides a very clear illustration of the spin-charge separation phenomenon known to exist in the 1D Hubbard model. Upon doping, the relevant charge excitation is not the quasiparticle excitation (spin-polaron) which carries both the spin and the charge of the doping hole, but rather a deconfined pair excitation consisting of a charged bosonic domain-wall (carrying charge but no spin) and a neutral fermionic domain-wall (carrying spin but no charge).

Another interesting result of this analysis is that the excitation energy needed to add a hole to an even-site chain is different from that needed to add a hole to an odd-site chain (see Fig.17, circles and diamonds). Such a difference would be irreconcilable if the excitation was a local quasiparticle, in which case different boundary conditions are expected to introduce variations of the order $\mathcal{O}(1 / N)$. However, in the presence of the charge-spin separation, there is a simple interpretation. A doped chain with an odd $N$ number of sites has an even $N-1$ number of electrons. Its ground state has zero spin (all electrons are paired). This is well described by a single charged domain-wall. On the other hand, the doped chain with an even $N$ number of sites has an odd $N-1$ number of electrons. In order to represent the total spin $1 / 2$ of the unpaired electron, it is necessary to include both a charged domain wall and a neutral spin- $1 / 2$ soliton. We associate the difference $\Delta E$ between the excitation energy of a single hole on odd and even-site chains with the energy of the additional neutral spin- $1 / 2$ soliton. This interpretation may be verified independently by evaluating the energy of a single neutral spin- $1 / 2$ domain wall on an odd-site, undoped chain. The BA ground-state energy of the undoped odd-site chain varies with the odd$N$ number of sites as $E(N)=N e_{G S}+E_{d w}$, where $e_{G S}$, the undoped ground-state energy per site, has the same value as obtained from fits of even-site chains. We identify $E_{d w}$ as the energy of the neutral spin soliton. The energies $E_{d w}$ and $\Delta E$ are compared in Fig.18. The good agreement between these independent measures of energy confirms that the spin soliton is a well defined concept even in the presence of doping. The existence of the spin soliton accounts for the difference in energy between odd and even site chains even in the thermodynamic limit. It facilitates our identification of the spin soliton in the Bethe ansatz with the neutral spin-1/2 domain wall of the CI approximation.

\section{CONCLUSIONS}

In this paper we have demonstrated the validity of the Configuration Interaction Method as a technique for going beyond mean field theory in strongly correlated electron systems. The method recaptures the essential physical features as well as energetics of the exact solution of the 1D Hubbard chain. The CI method provides a systematic way to improve and go beyond the Hartree-Fock Approximation, by incorporating essential quantum dynamics and tunneling effects which are excluded from the mean-field theory.

We showed that a charged bosonic domain wall can lower its kinetic energy by about $t$ for all $U / t$ values, 
while the immobile charged spin-polaron can only lower its kinetic energy by an energy of the order of $t^{2} / U$. As a result, the mobile charged bosonic domain wall is the low energy charged excitation of the Hubbard chain for all values of $U / t$, and its excitation energy and dispersion band are in good agreement with the predictions of the exact Bethe-Ansatz solution. We also showed that it is energetically favorable for the (quasiparticle-like) charged fermionic spin-bag to dissociate into a charged bosonic domain wall (which carries the charge but no spin) and a neutral spin-1/2 domain wall (which carries the spin but no charge). Clearly the CI method recaptures the physics of spin-charge separation known to exist in the 1D Hubbard model. It may be fruitful to perform a more detailed study of the interaction between various types of domain walls, both charged and uncharged. Various correlation functions and response functions can also be calculated for detailed comparison with exact results.

Given the validity and accuracy of the Configuration Interaction Method for the 1D problem, we believe that it provides a natural route to describe effects beyond meanfield theory in the doped ground-state of the 2D Hubbard model and other models of strongly correlated electrons in higher dimensions. We present this study elsewhere.

\section{ACKNOWLEDGMENTS}

M.B. acknowledges support from the Ontario Graduate Scholarship Program and a fellowship from William F. McLean. This work was supported in part by the Natural Sciences and Engineering Research Council of Canada.

${ }^{1}$ P.W. Anderson, Science 235, 1196 (1987).

${ }^{2}$ E.H. Lieb and F.Y. Wu, Phys. Rev. Lett. 20, 1445 (1968).

${ }^{3}$ H. Bethe, Z. Phys. 71, 205 (1931); L. Hulthen, Ark. Mat. Astron. Fys., bf 26 A, No. 11 (1938).

${ }^{4}$ E. Louis, F. Guinea, M.P. Lopez Sancho and J.A. Verges, Phys. Rev. B 59, 14005 (1999).

${ }^{5} \mathrm{M}$. Berciu and S. John, to be published.

${ }^{6}$ S. John and A. Golubentsev, Phys. Rev. Lett. 71, 3343 (1993); S. John and A. Golubentsev, Phys. Rev. B 51, 381 (1995).

${ }^{7}$ Mona Berciu and Sajeev John, Phys. Rev. B 59, 15143 (1999).

8 T.A. Friedmann, M.W. Rabin, J. Giapintzakis, J.P. Rice and D.M. Ginsberg, Phys. Rev. B42, 6217 (1990); R. Gagnon, C. Lupien and L. Taillefer, Phys. Rev. B 50, 3458 (1994).

${ }^{9}$ D.N. Basov, R. Liang, D.A. Bonn, W.N. Hardy, B. Dabrowski, M. Quijada, D.B. Tanner, J.P. Rice, D.M. Ginsberg and T. Timusk, Phys. Rev. Lett. 74, 598 (1995).

${ }^{10}$ K. Zhang, D.A. Bonn, S. Kamal, R. Liang, D.J. Baar, W.N.
Hardy, D. Basov and T. Timusk, Phys. Rev. Lett. 73, 2484 (1994).

11 J.L. Tallon, C. Bernhard, U. Binninger, A. Hofer, G.V.M. Williams, E.J. Ansaldo, J.I. Budnick and Ch. Niedermayer, Phys. Rev. Lett 741008 (1995).

${ }^{12}$ P. Fulde, Electron Correlations in Molecules and Solids, (Springer, 1995).

13 Z.N.C. Ha, Quantum Many-Body Systems in One Dimension, (World Scientific, 1996).

${ }^{14}$ D.C. Mattis, The Theory of magnetism I, (Springer, 1981).

${ }^{15}$ M. Berciu and S. John, Phys. Rev. B 57, 9521 (1998).

${ }^{16}$ P.W. Anderson, Phys. Rev. 115, 2 (1959).

17 A.J. Heeger, S. Kivelson, J.R. Schrieffer and W.-P. Su, Rev. Mod. Phys. 60, 781 (1988).

${ }^{18}$ W.-P. Su, J.R. Schrieffer and A.J. Heeger, Phys. Rev. B 22, 2099 (1980).

FIG. 1. Ground-state energy per site, in units of $t$, of an undoped Hubbard chain of $N$ sites and $U / t=5$. The full circles show the values found directly from the Bethe Ansatz Equations (15, 16, 17) while the full line shows the thermodynamic limit given by Eq. (18). In the limit of large $N$ the two values agree.

FIG. 2. Energy per site (in units of $t$ ) of the AFM undoped background as a function of $U / t$, as obtained from the Hartree-Fock Approximation (full line) and from the exact Bethe Ansatz solution (dashed line).

FIG. 3. Self-consistent spin and charge distribution for a 40-site chain with two neutral domain walls, for $\mathrm{U} / \mathrm{t}=5$. The charge $Q(i)=1$ everywhere. The total spin carried by each neutral domain wall is $1 / 2$.

FIG. 4. Electronic structure of the 40-site chain with the two neutral domain walls shown in Fig. 3. Each domain wall has 4 discrete levels bound in its core. The spins on the two occupied bound levels are oriented in the same direction as the core spins of the domain wall. In the configuration shown in Fig. 3 , there is a $+1 / 2$ and $\mathrm{a}-1 / 2$ domain wall, and therefore all levels are spin paired and the total spin of the chain is zero. However, for two $+1 / 2(-1 / 2)$ domain walls, all the occupied discrete levels have $+1 / 2(-1 / 2)$ spins, and the total spin of the chain is $+1(-1)$.

FIG. 5. Ground-state energy (in units of $t$ ) of a chain of size $N$, calculated with the HF (circles), CI with two neutral domain walls (squares) and BA (diamonds). The left panel corresponds to $U / t=5$, while the right one corresponds to $U / t=50$. Although the energy scale is very different, in both cases the CI method significantly improves the agreement with the exact Bethe Ansatz solution. 
FIG. 6. Self-consistent charge (upper line) and spin distributions for a charged spin-bag on a 40 -site chain, for $U / t=5$. The spin-bag is a charged fermion.

FIG. 7. Self-consistent charge (upper line) and spin distributions for a domain wall centered on site (COS) on a 41-site chain, for $U / t=5$.

FIG. 8. Self-consistent charge (upper line) and spin distributions for a domain wall centered between sites (CBS) on a 41-site chain, for $U / t=5$. The domain walls are charged bosons.

FIG. 9. Electronic spectra for an undoped AFM chain with 40 sites (left panel), and for a 40-site chain with a charged spin-bag (right panel). $U / t=5$.

FIG. 10. Electronic spectra for a chain 41 sites chain with a CBS domain-wall (left panel), and a COS domain-wall (right panel). $U / t=5$.

FIG. 11. Excitation energy in units of $t$ of the charged spin-polaron, CBS and COS domain walls, as a function of $U / t$. The excitation energy is defined with respect to the undoped chain. Within the HFA, for $U / t<6.5$ the charged domain-walls are the low-energy charged excitations, while for $U / t>6.5$ the spin-polarons are the low-energy excitations. However, the HFA approximation neglects the kinetic energy gained by these charged excitations through translation along the chain. When this is taken into account within the Configuration Interaction approximation, the mobile domain-wall is found to be the low-energy charged excitation for all values of $U / t$ (see Fig. 15).

FIG. 12. Dispersion band for the spin-polaron, $E_{\text {pol }}(k)$ vs. $k$, with $E_{\text {pol }}(k)$ extracted from Eq. (24) for chains of length $2 N=14,16, \ldots, 22$ sites and $U / t=5$. Also shown is the excitation energy of the static hole-doped spin-polaron $E_{\text {pol }}^{H F}$ (the full line), as obtained from the Unrestricted HFA. Translation lowers the total energy of the spin-polaron, with the most stable state corresponding to $k=\pi / 2 a$. However, the kinetic energy gained through translation is quite small.

FIG. 13. The kinetic energy gained by the delocalized spin-polaron $E_{\text {pol }}\left(\frac{\pi}{2 a}\right)-E_{\text {pol }}^{H F}$ (circles) and the width of the spin-polaron band, $E_{\text {pol }}\left(\frac{\pi}{2 a}\right)-E_{\text {pol }}(0)$ (squares) as a function of $t^{2} / U$. The log-log graph is linear with a slope of unity as expected. The spin-polaron must tunnel two sites to the next allowed position, through a second order hopping process. This charged fermion is rather immobile.
FIG. 14. The dispersion relations $E_{d w}(k)$ vs. $k$ for both CBS (left panel) and COS (right panel) domain walls on chains of different length $2 N+1=17, \ldots, 23$, and $U / t=5$. The excitation energy of the static configuration (obtained from the unrestricted HF search) is also shown as a full line. The extra kinetic energy gained through translation by the domain-wall is of the order of $t$.

FIG. 15. Excitation energy, in units of $t$, for a mobile charged domain wall (circles) and a mobile charged spin-polaron (diamonds), as obtained from the CI approach. The exact excitation energy given by the Bethe-Ansatz method is shown by squares. The domain-wall CI energy is in excellent agreement with the exact BA results (also see inset), while the spin-polaron CI energy is significantly different. For comparison, we also show the excitation energies for the COS and CBS domain walls as obtained from the static HFA (up and down triangles), proving again that the extra kinetic energy gained by the moving domain wall is of order $t$ for most $U / t$ values. In contrast, the extra kinetic energy gained by the spin-polaron is of order $t^{2} / U \rightarrow 0$ as $U / t$ increases, so in the large $U / t$ limit there is almost no difference between the $\mathrm{HF}$ and CI results for the charged spin-polaron. We conclude that the charged domain-wall is the relevant excitation for all values of $U / t$.

FIG. 16. The dispersion relation for a domain-wall on a chain of length $2 N+1=17,19,21$ and $U / t=100$. In the large $U / t$ limit the dispersien relation of one single hole is given by $E_{d w}(k)=2 t \cos (k a) .44$ This is indeed in very good agreement with the dispersion band of the domain wall, proving again that this is the relevant charged excitation of the Hubbard chain.

FIG. 17. Excitation energy (in units of $t$ ) for a charged domain-wall - neutral domain-wall pair, as obtained from the CI approach for even-site chains (squares). The energy of the doping hole added to an even-site chain, as obtained from the exact Bethe Ansatz solution, is shown by circles. Again, there is good agreement between the two methods. For comparison, we also show the energy of the doping hole added to an odd-site chain, as obtained from the Bethe Ansatz (diamonds) and the energy of an isolated charged domain wall on odd-site chains (triangles). These last two sets are the same as in Fig.15.

FIG. 18. The excitation energy of a neutral domain-wall, as obtained from the Bethe ansatz for undoped odd-site chains (circles). This agrees with (diamonds) the difference in the excitation energy of a single hole on an even-site vs. odd-site chain (shown individually as circles and diamonds of Fig.17). The dotted line is the asymptotic fit $6 t^{2} / U$. 
Figures for manuscript "Quantum dynamics of charged and neutral magnetic solitons: Spin-charge separation in the one-dimensional Hubbard model" by Mona Berciu and Sajeev John

FIG. 1

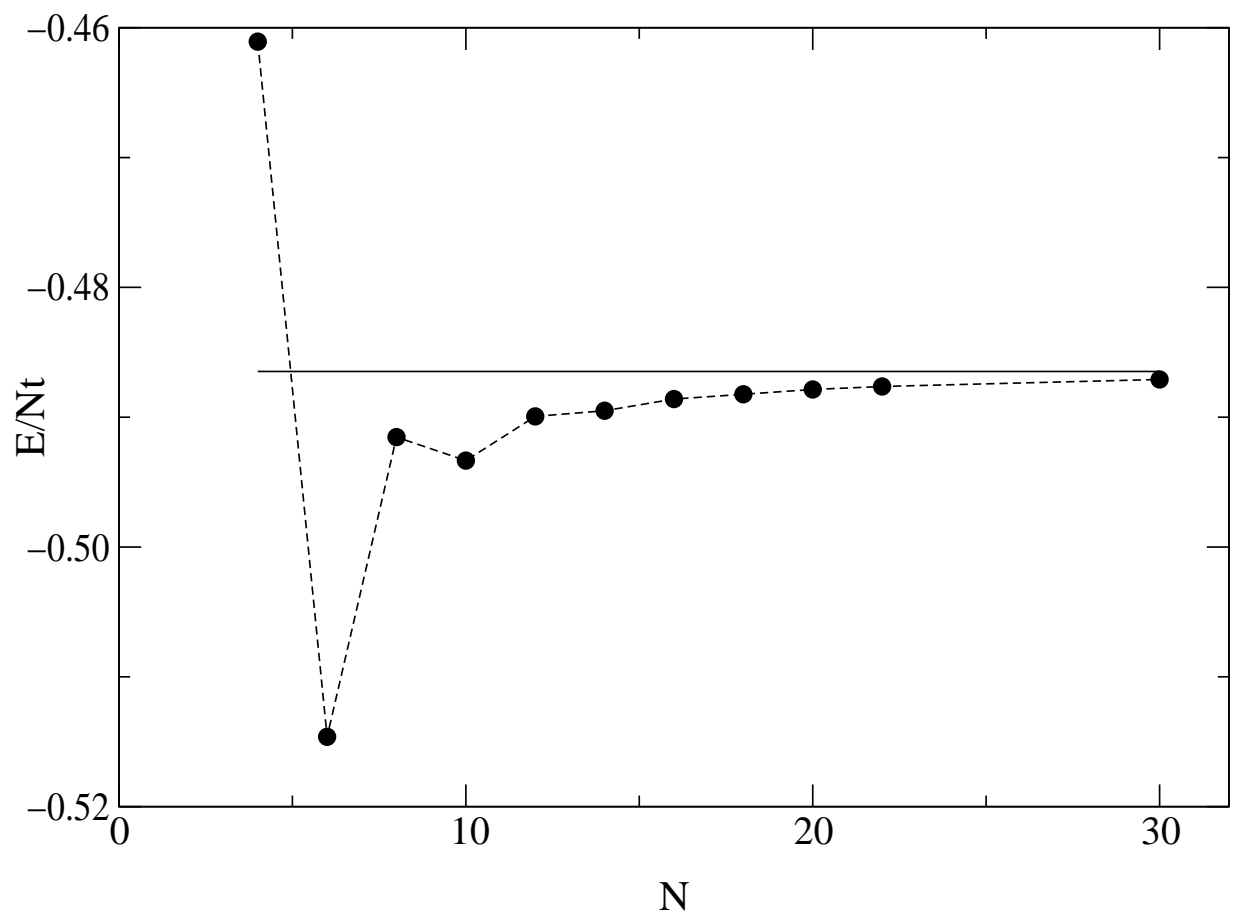

FIG. 2

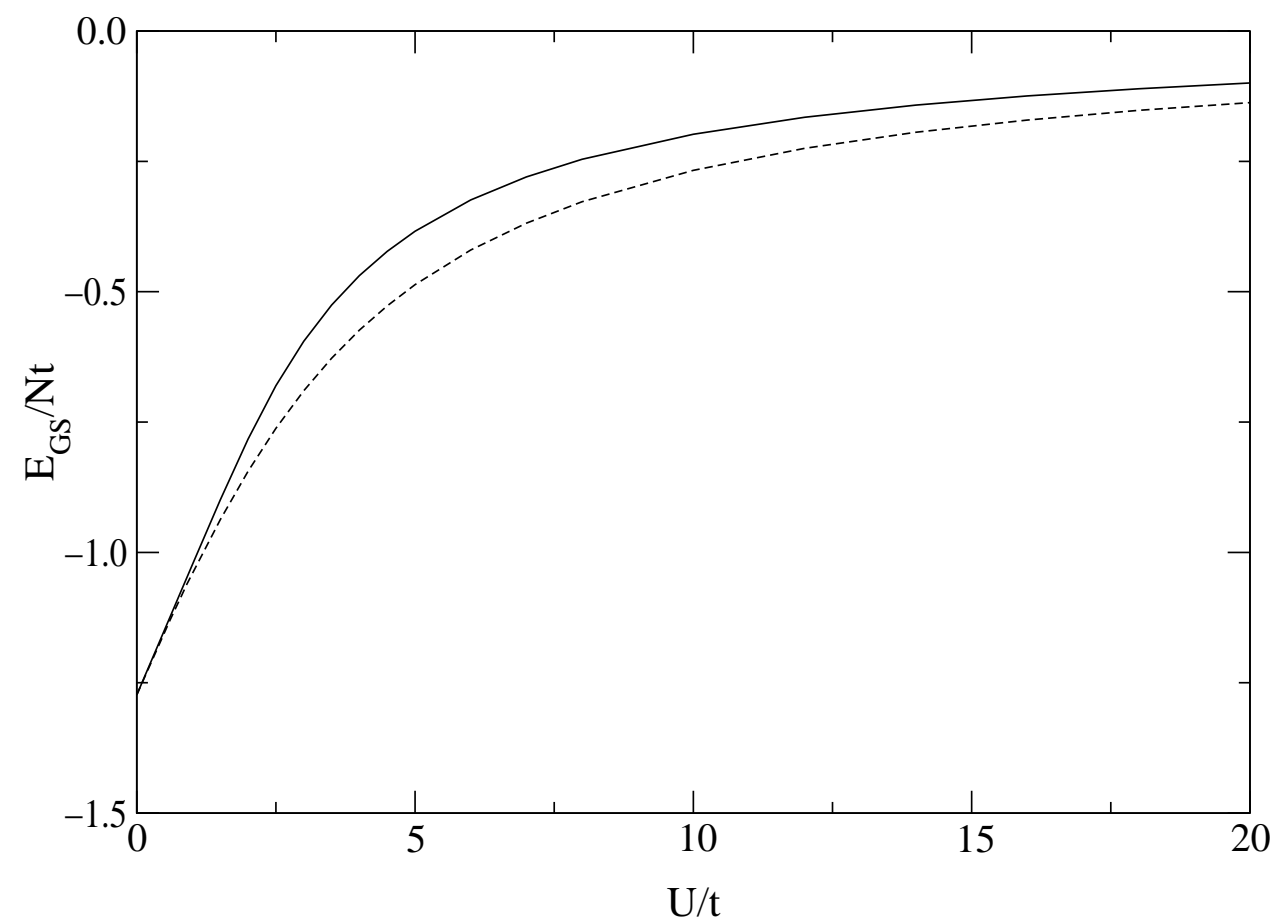


- M. Berciu and S. John: Quantum dynamics of charged and neutral magnetic solitons:Spin-charge separation in the one

FIG. 3

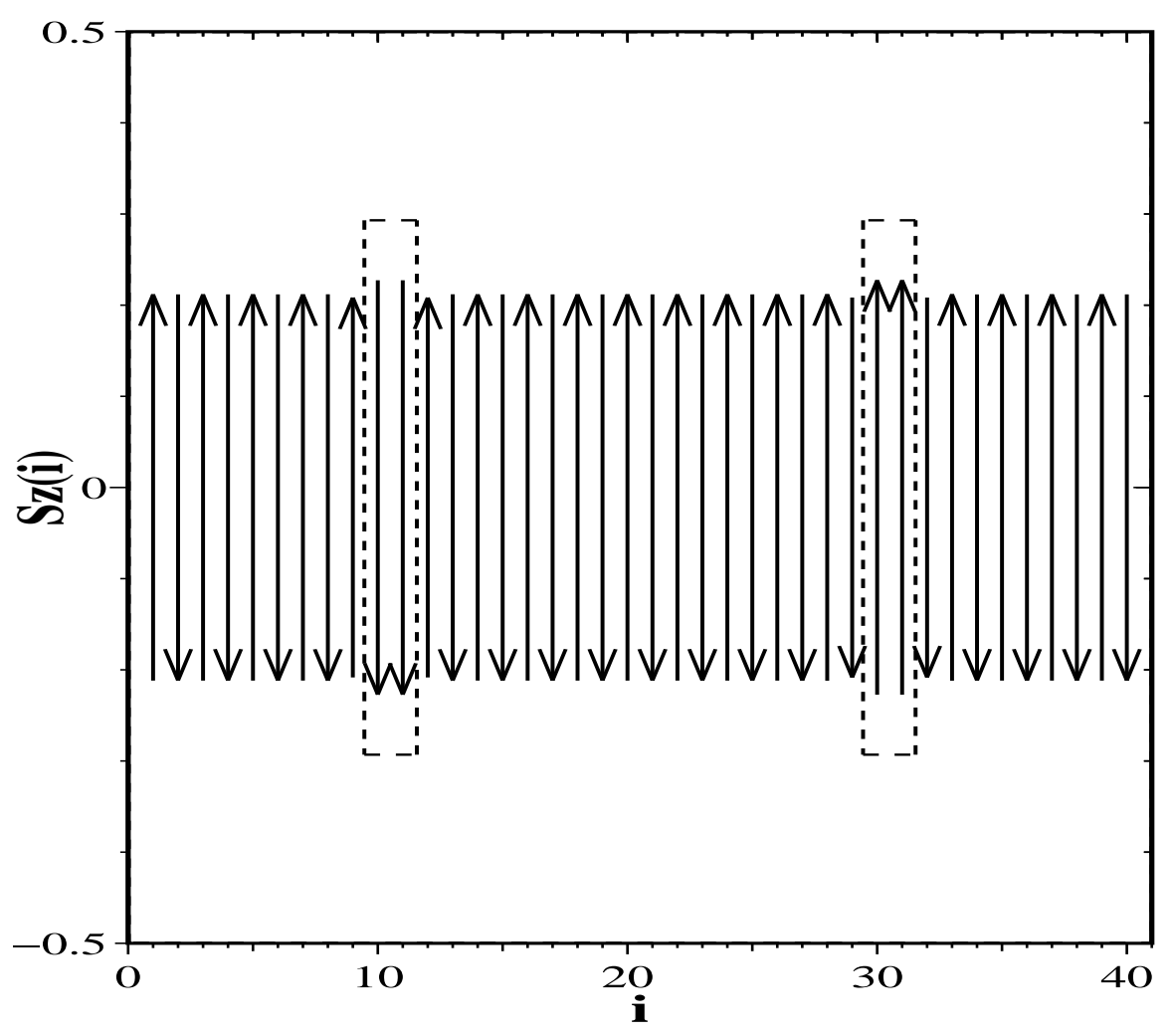

FIG. 4

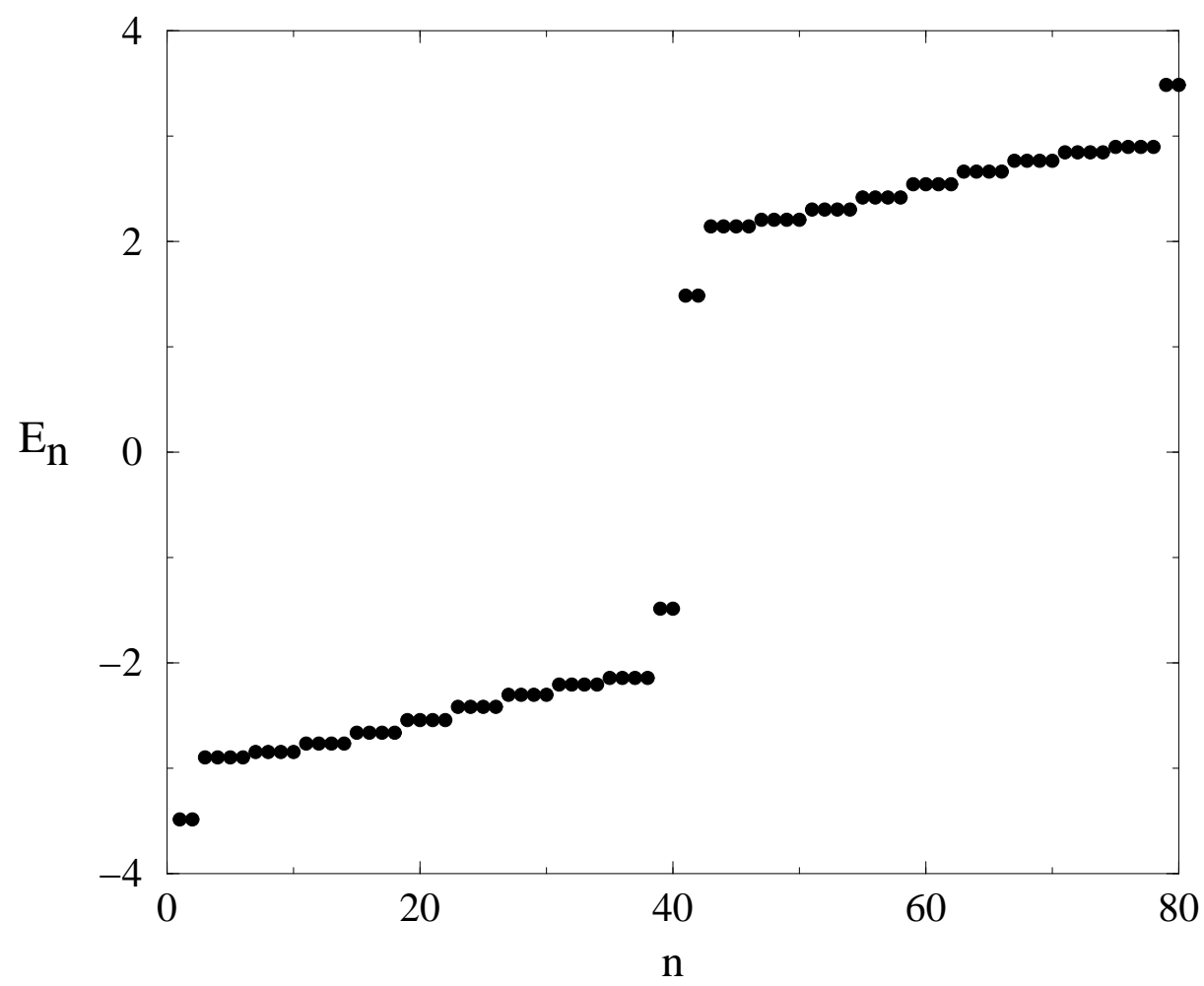


- M. Berciu and S. John: Quantum dynamics of charged and neutral magnetic solitons:Spin-charge separation in the one

FIG. 5

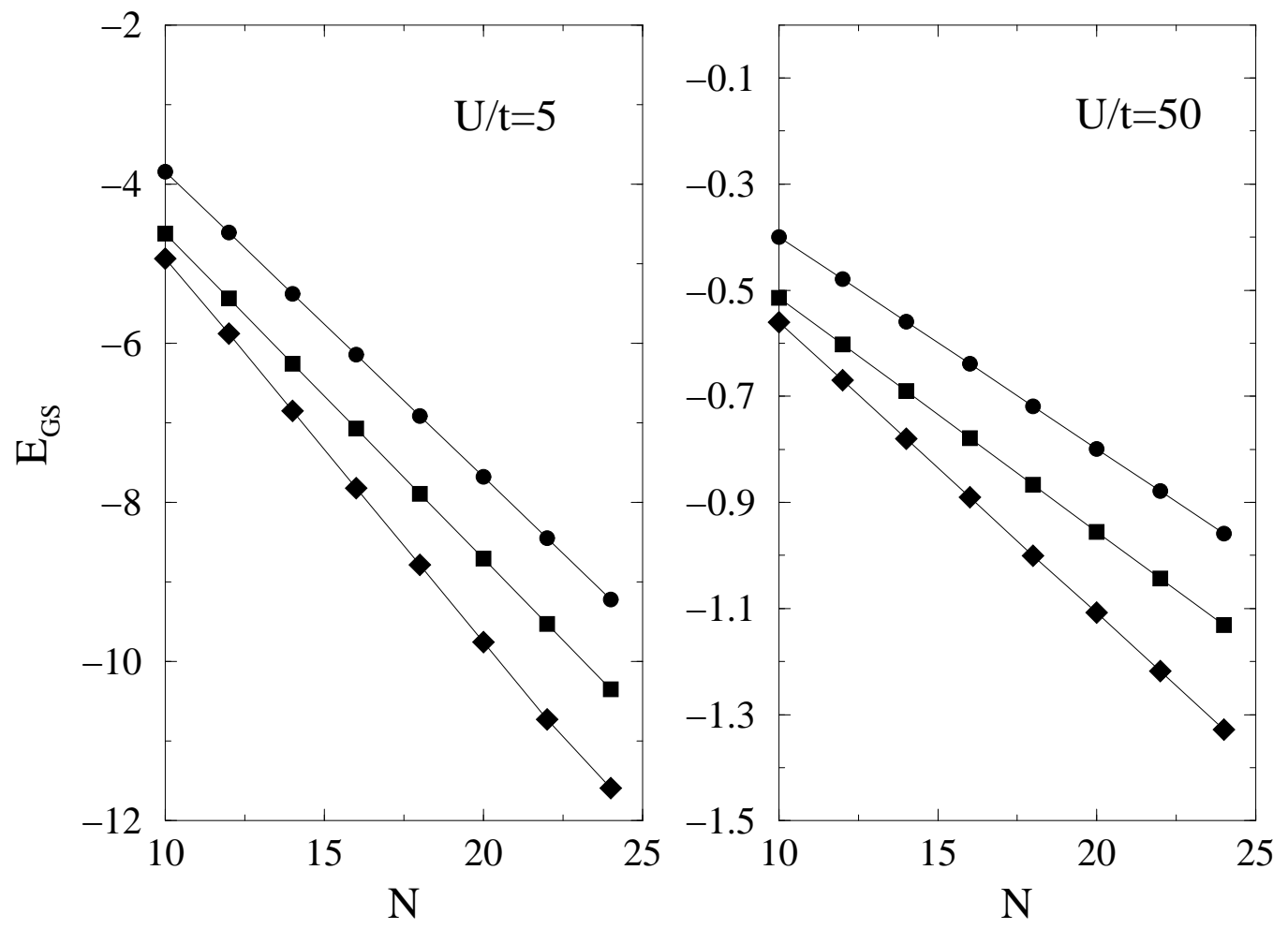

FIG. 6

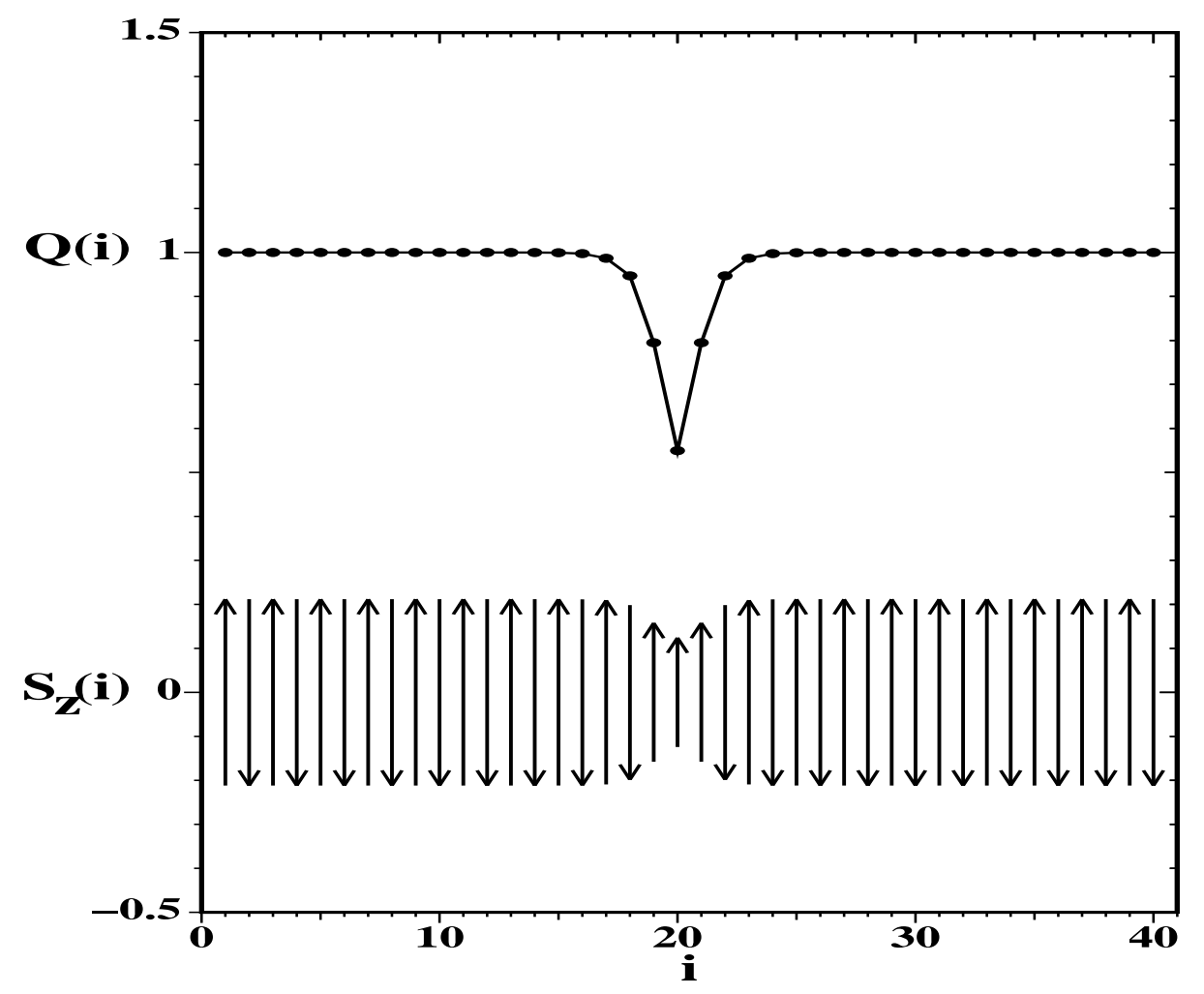


- M. Berciu and S. John: Quantum dynamics of charged and neutral magnetic solitons:Spin-charge separation in the one

FIG. 7

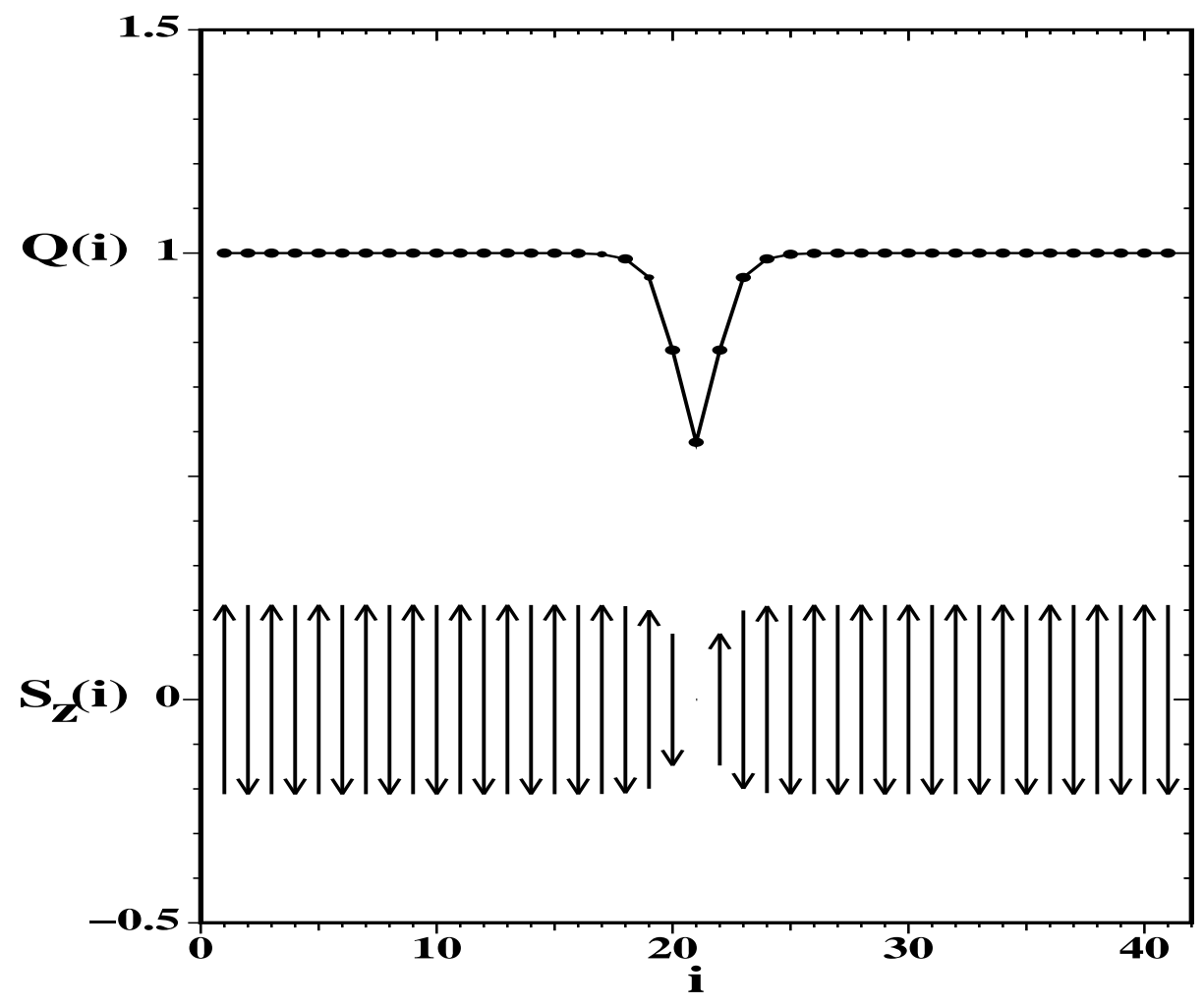

FIG. 8

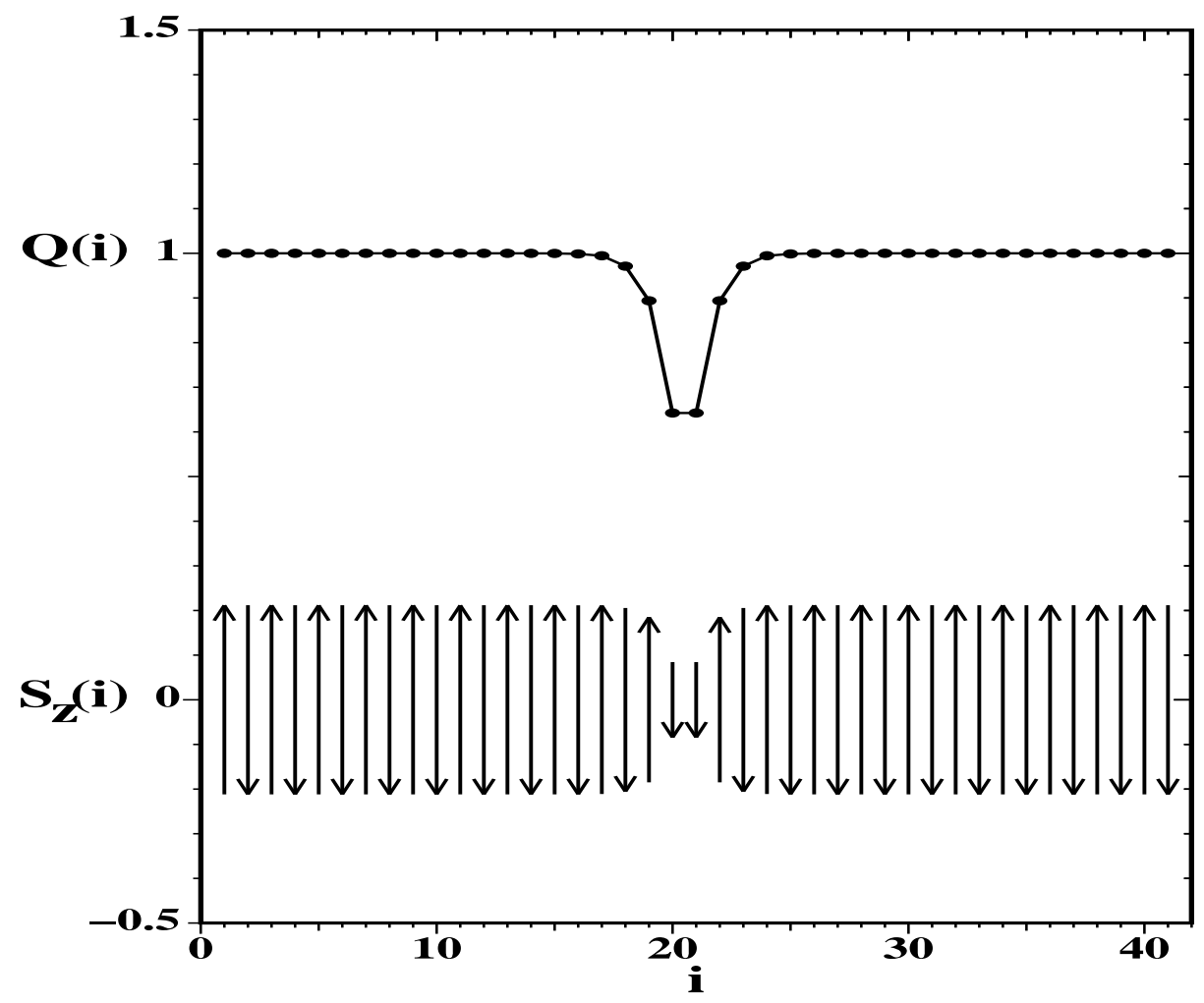


- M. Berciu and S. John: Quantum dynamics of charged and neutral magnetic solitons:Spin-charge separation in the one

FIG. 9
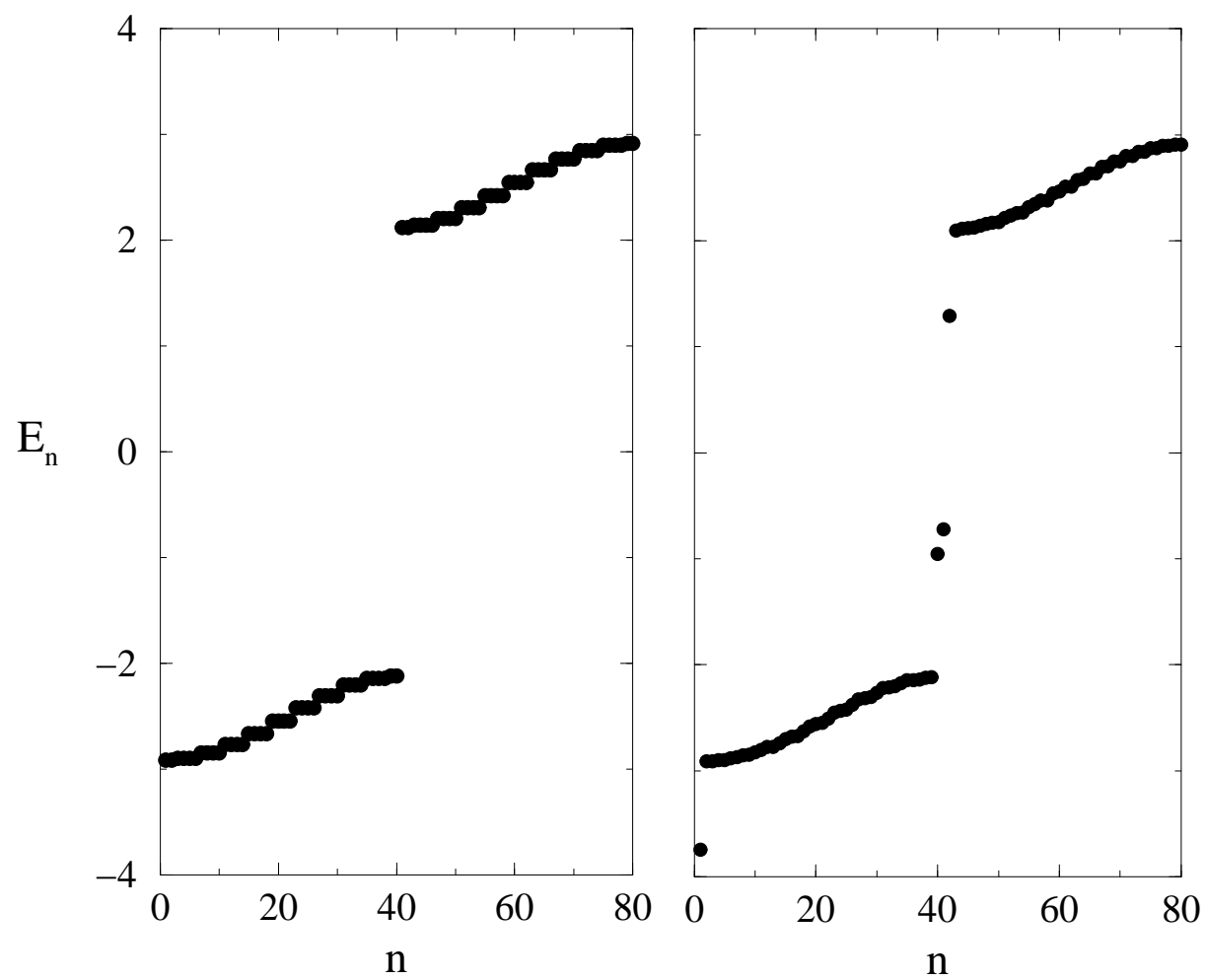

FIG. 10
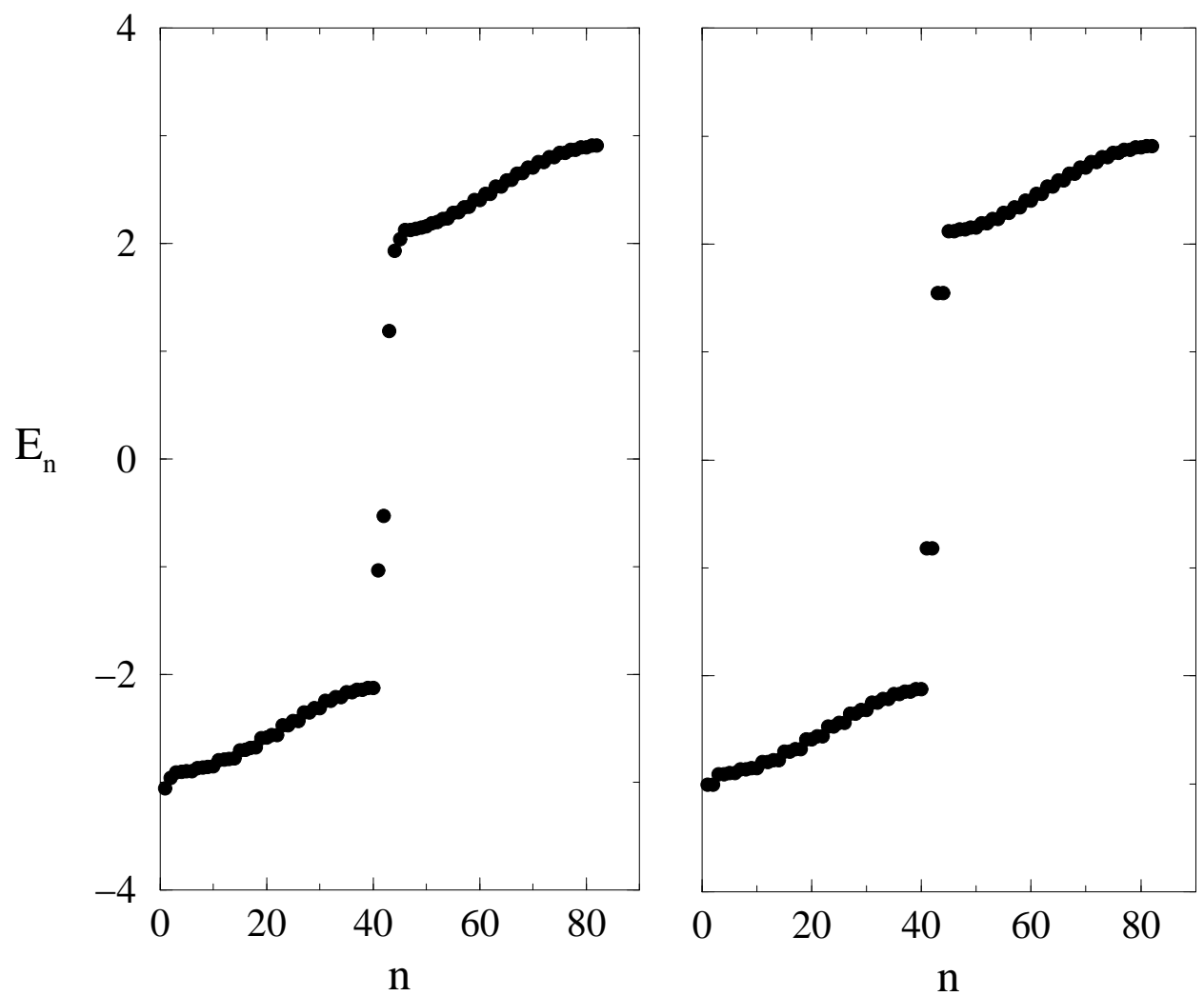
- M. Berciu and S. John: Quantum dynamics of charged and neutral magnetic solitons:Spin-charge separation in the one

FIG. 11

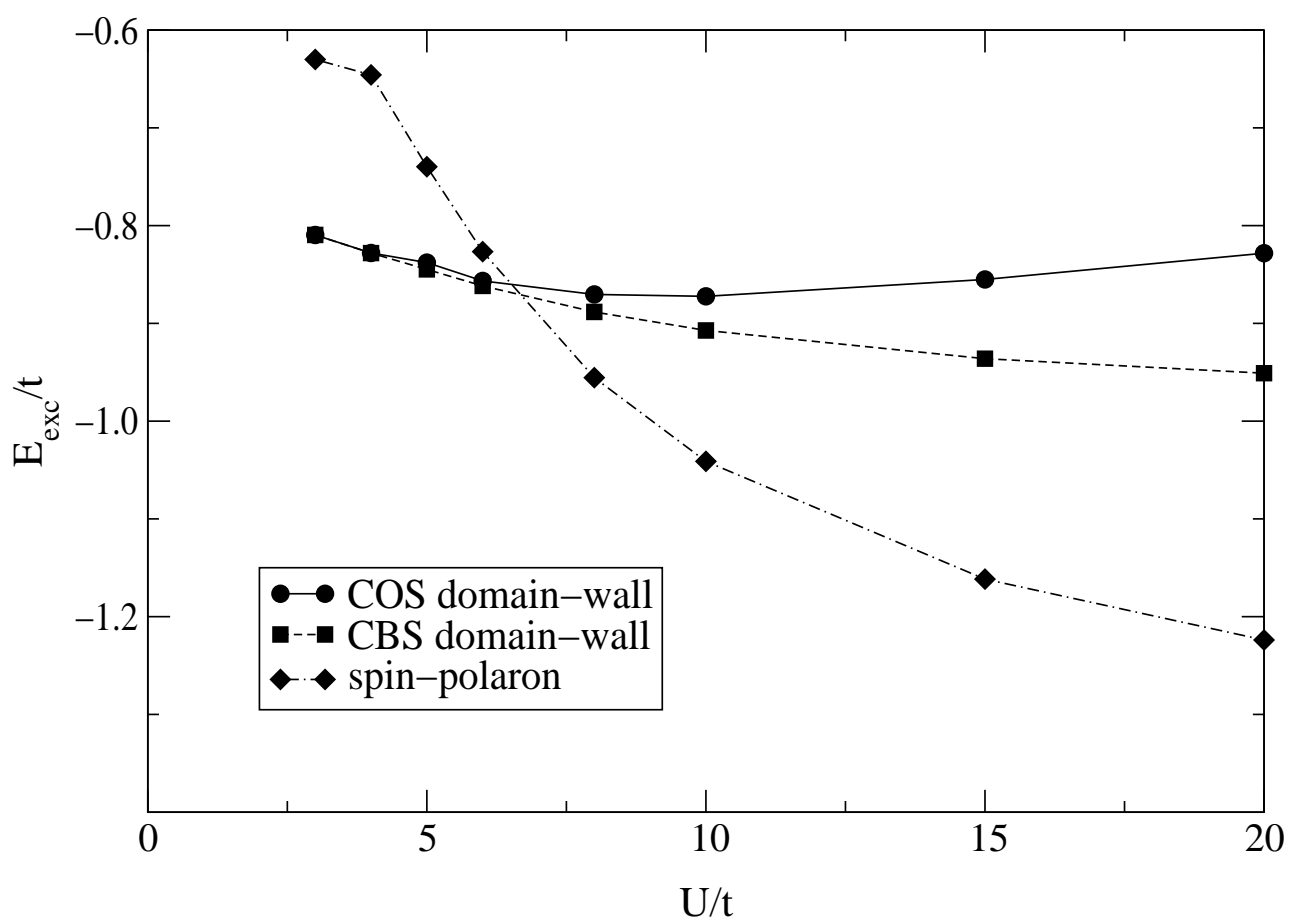

FIG. 12

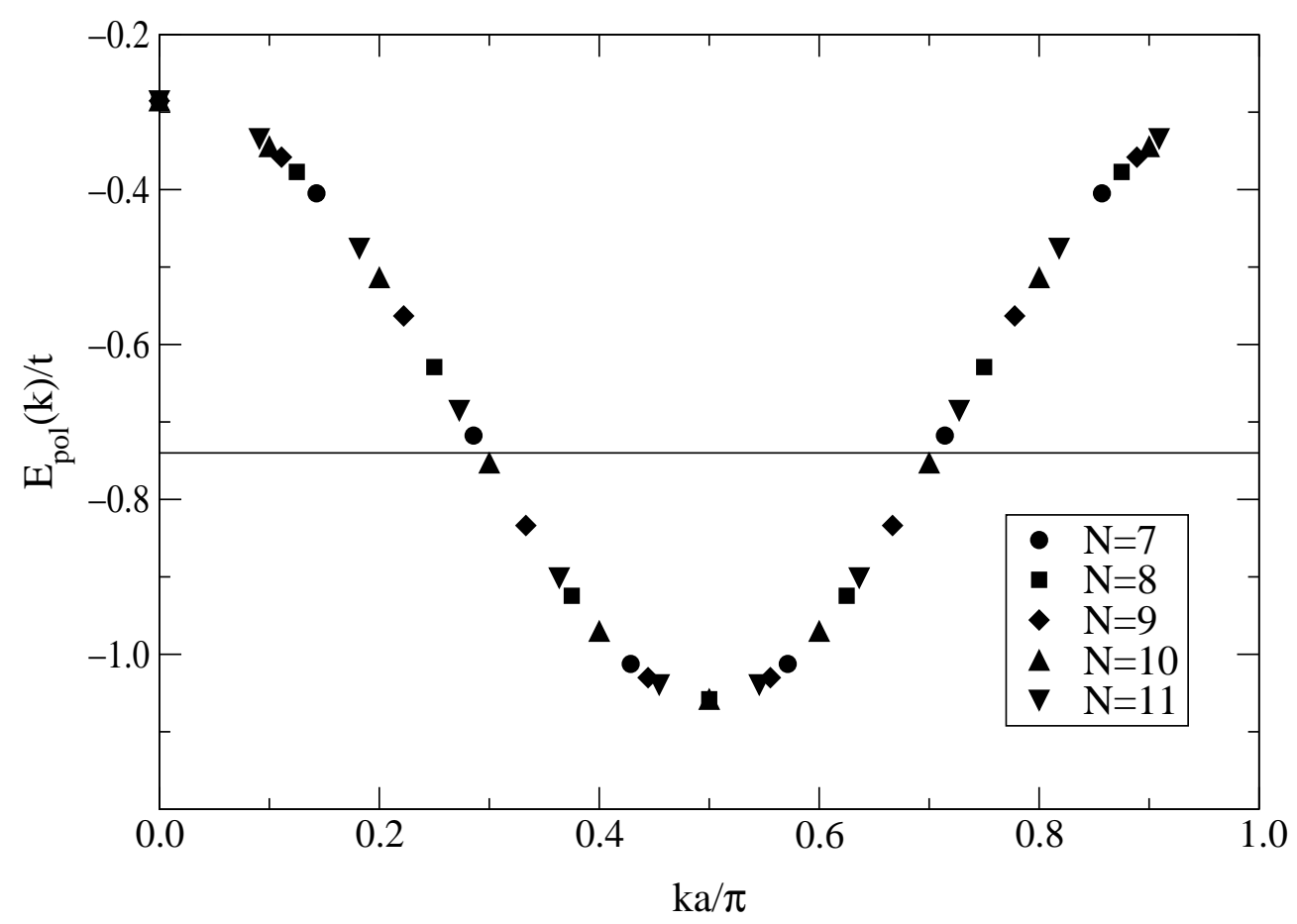


- M. Berciu and S. John: Quantum dynamics of charged and neutral magnetic solitons:Spin-charge separation in the one

FIG. 13

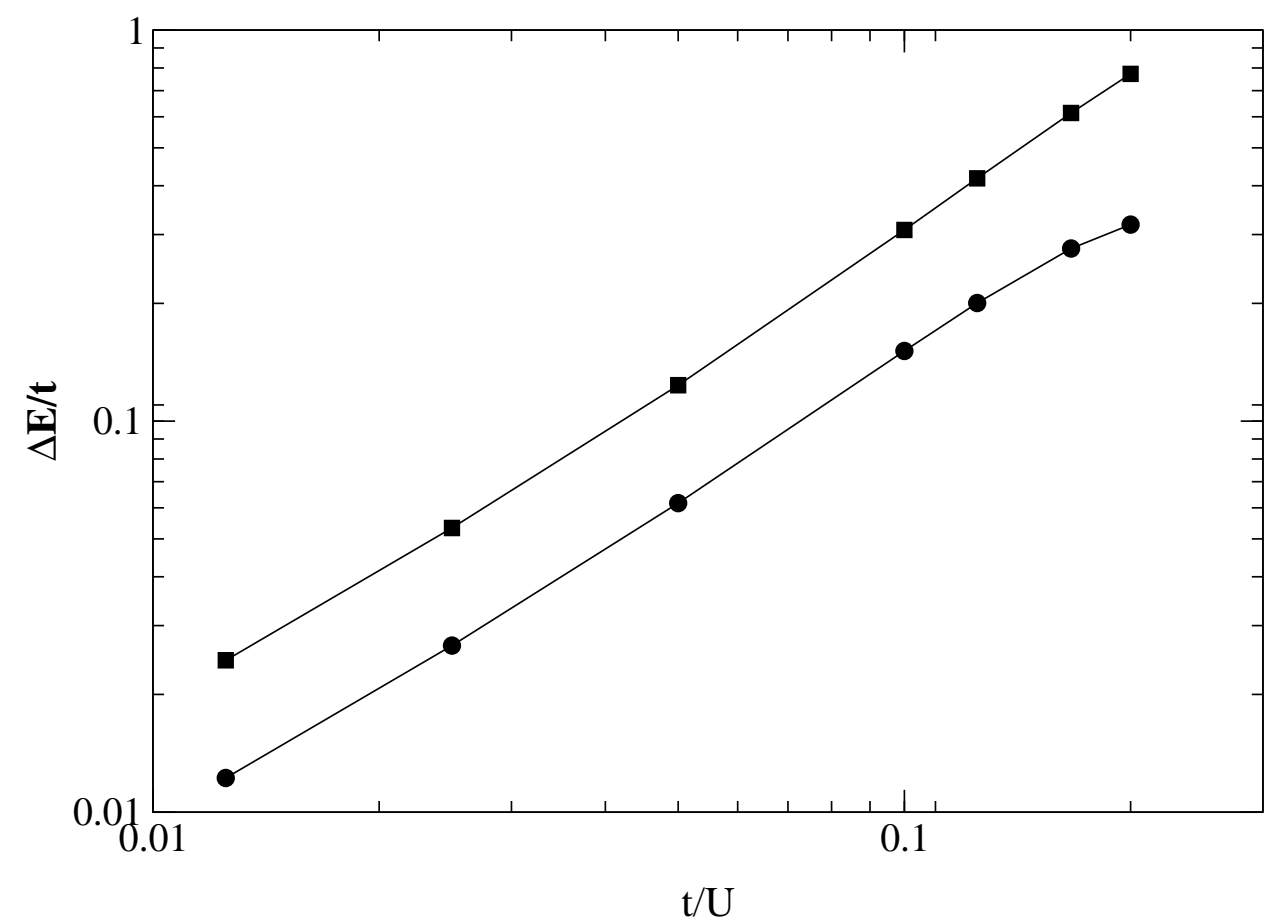

FIG. 14
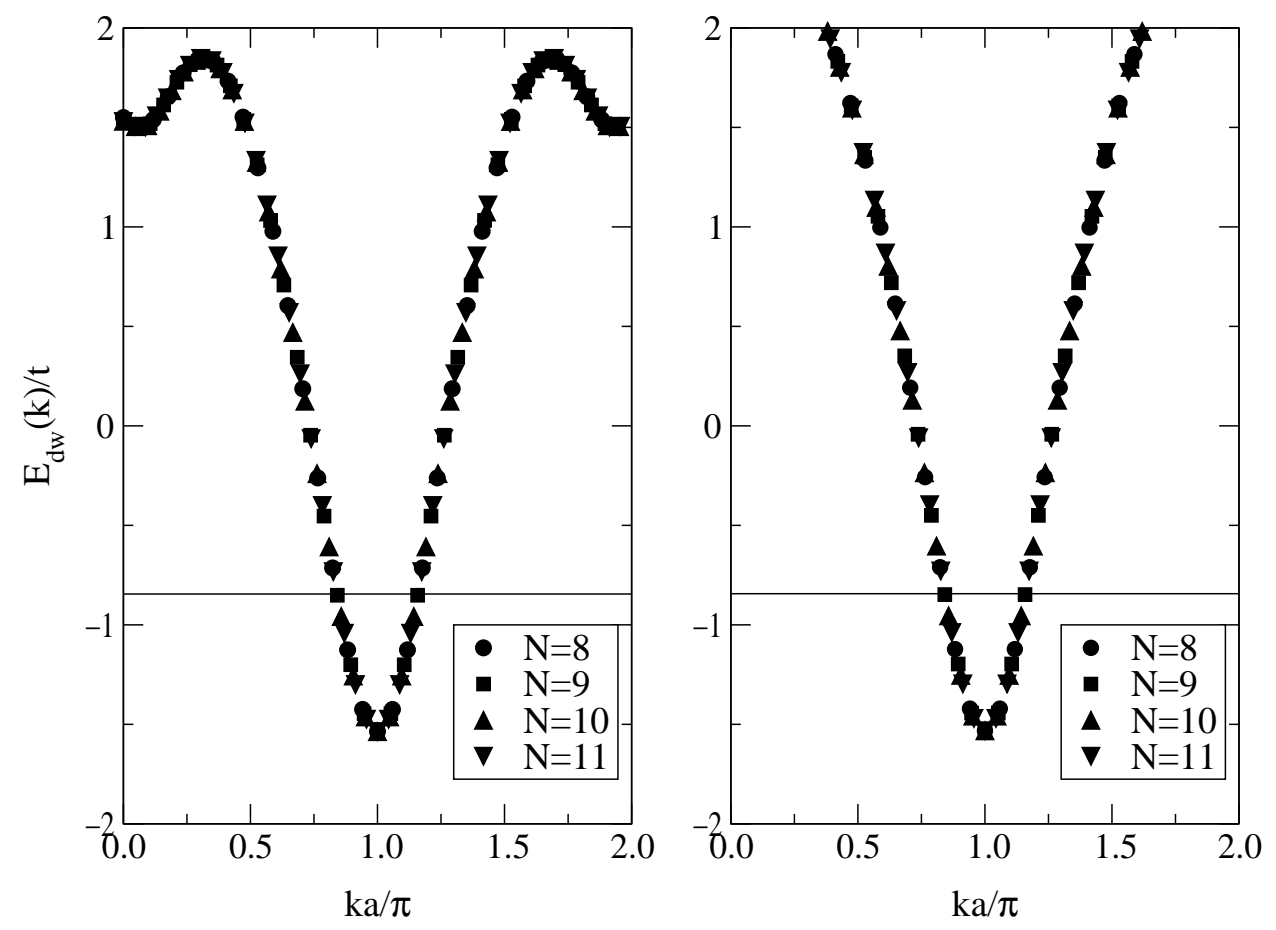
- M. Berciu and S. John: Quantum dynamics of charged and neutral magnetic solitons:Spin-charge separation in the one

FIG. 15

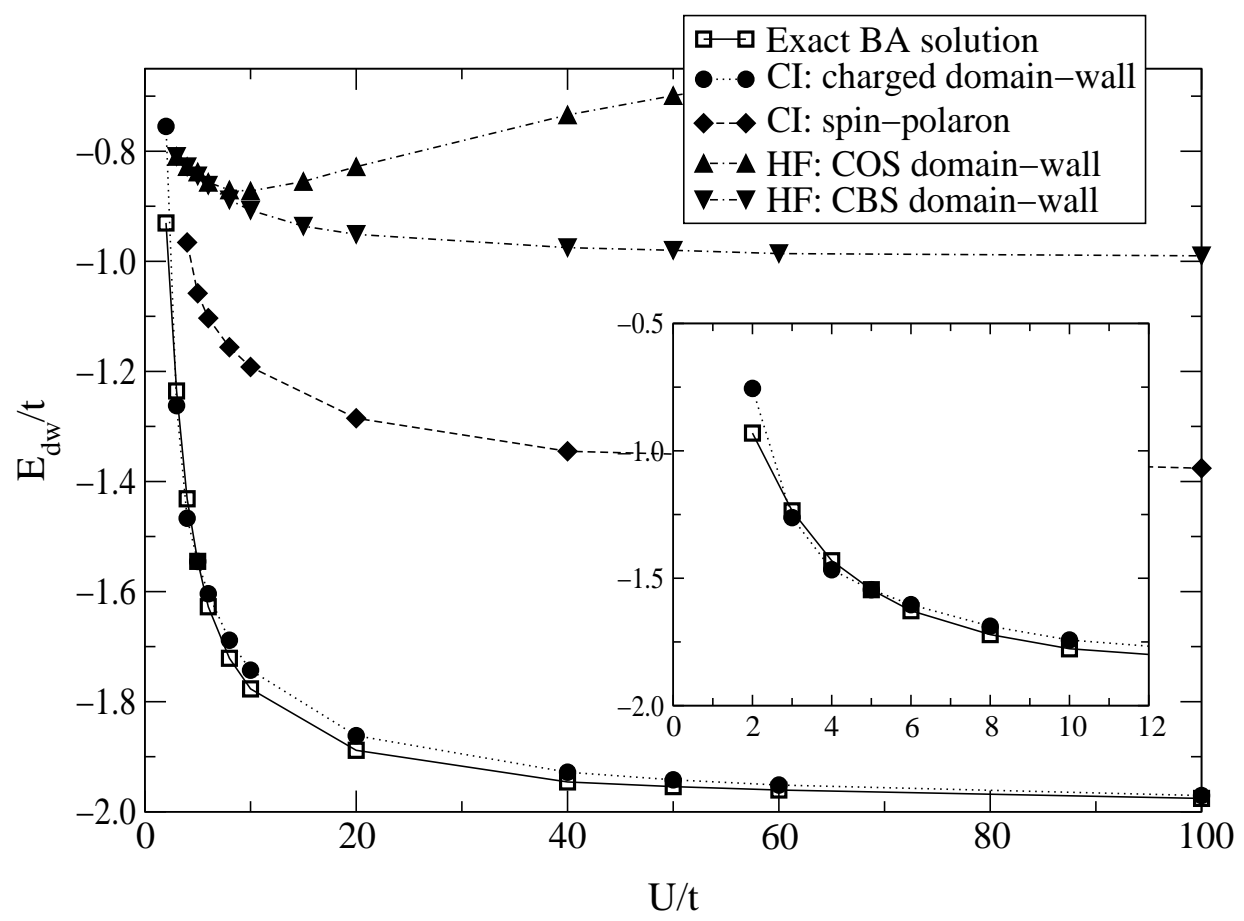

FIG. 16

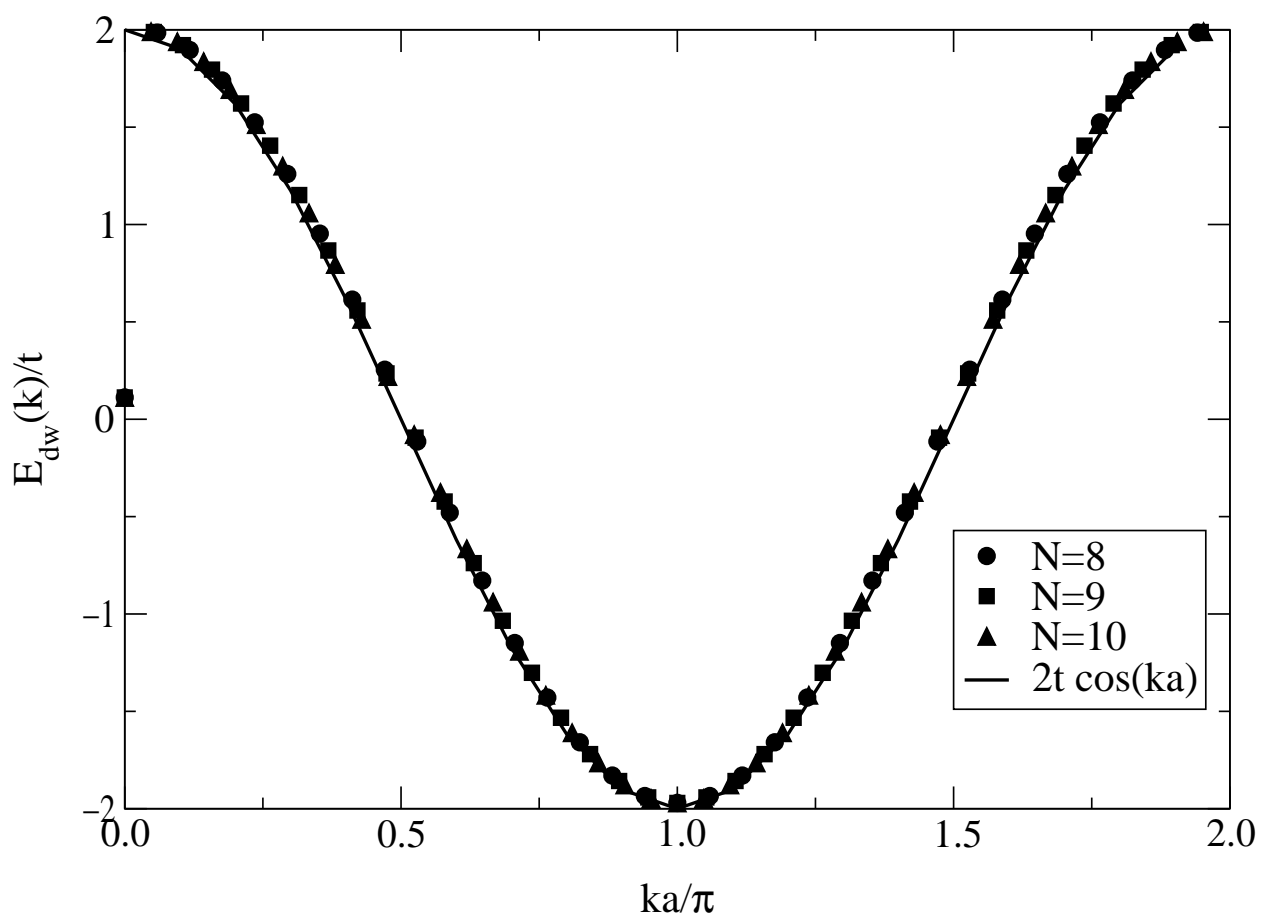


- M. Berciu and S. John: Quantum dynamics of charged and neutral magnetic solitons:Spin-charge separation in the one

FIG. 17

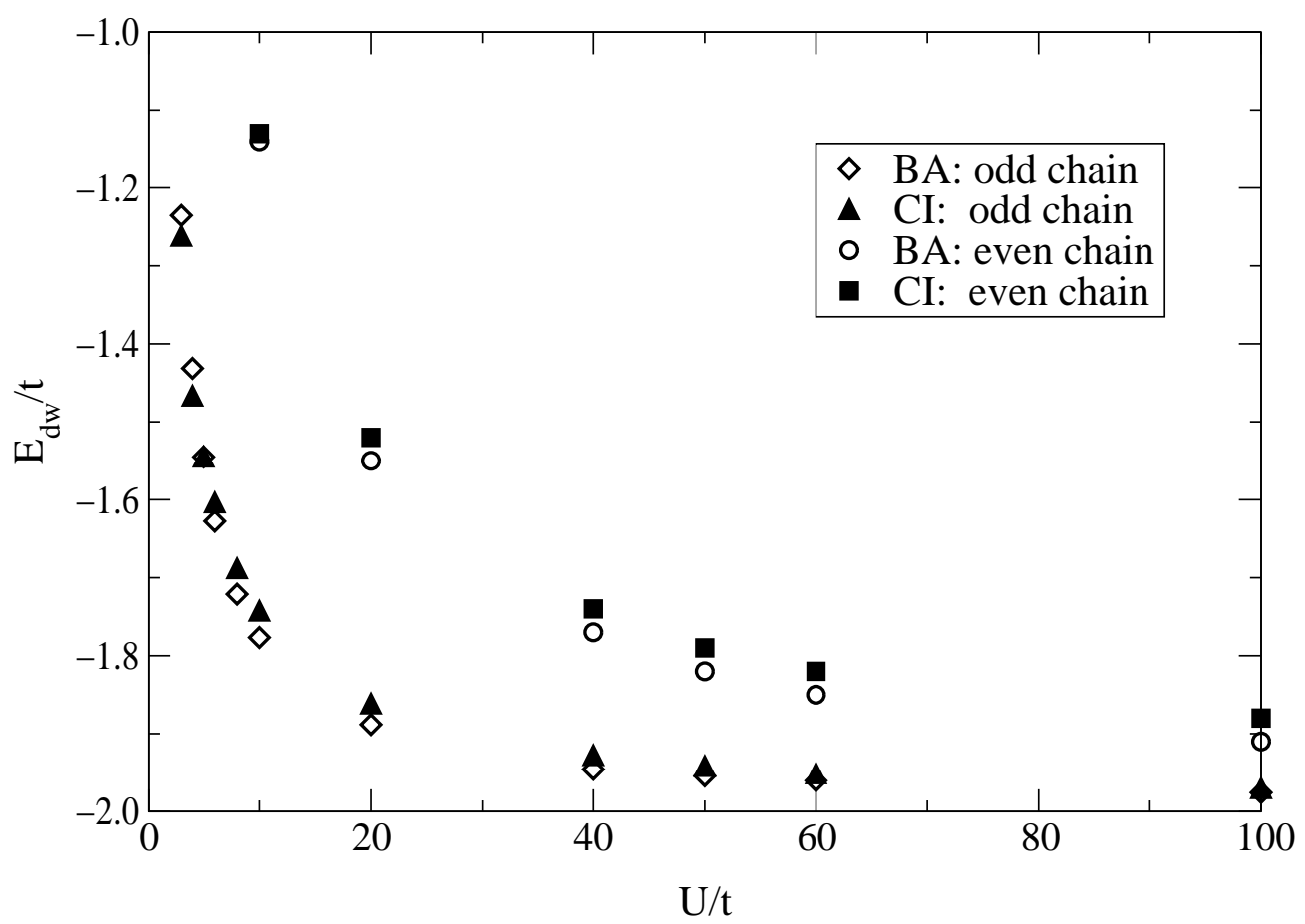

FIG. 18

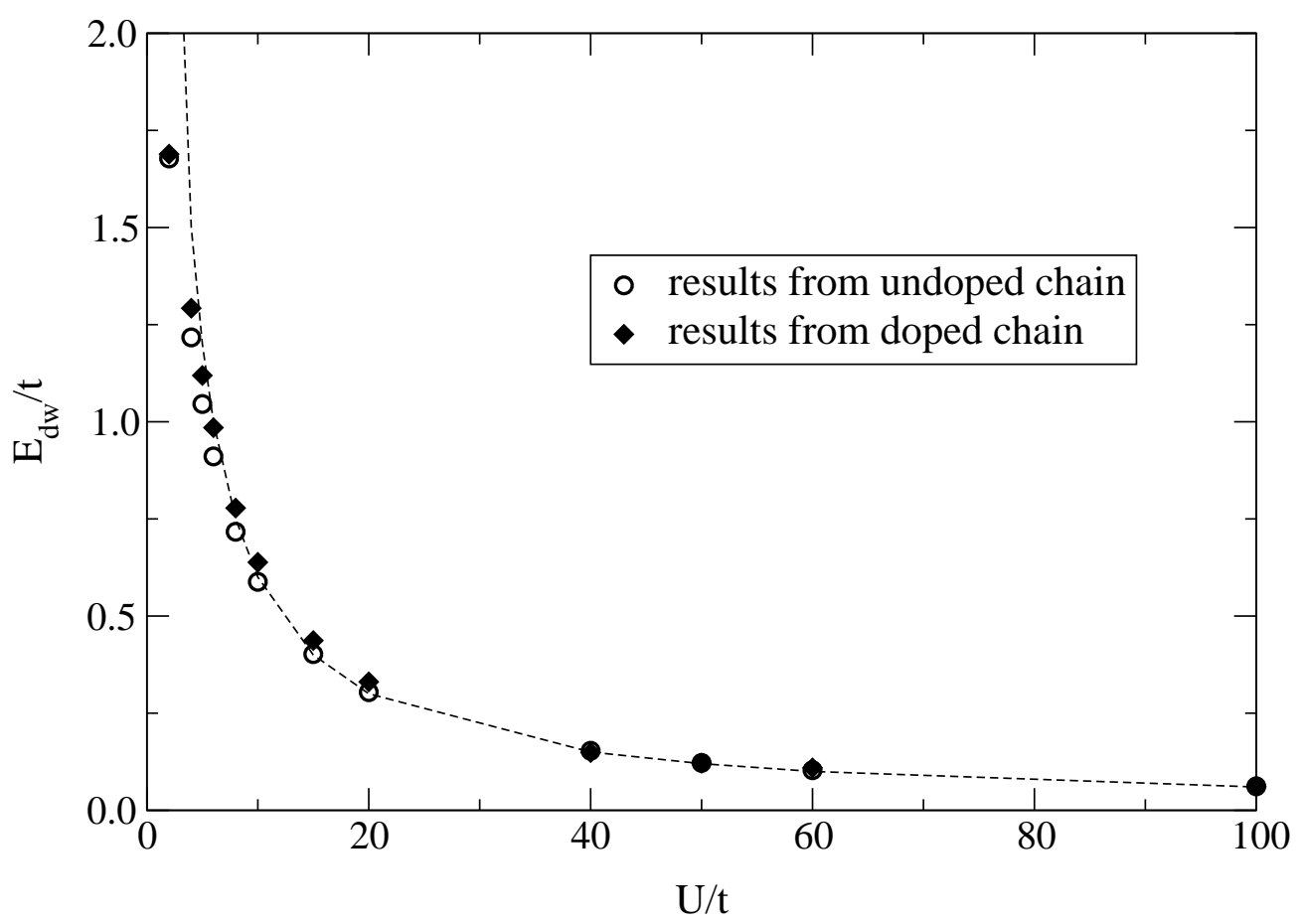

